\title{
TIDAL DISRUPTIONS OF WHITE DWARFS FROM ULTRA-CLOSE ENCOUNTERS WITH INTERMEDIATE-MASS SPINNING BLACK HOLES
}

\author{
Roland Haas $^{1,2}$, Roman V. ShCherbakov ${ }^{3,4}$, Tanja Bode ${ }^{1}$, and Pablo Laguna ${ }^{1}$ \\ ${ }^{1}$ Center for Relativistic Astrophysics, School of Physics, Georgia Institute of Technology, Atlanta, GA 30332, USA \\ ${ }^{2}$ Theoretical AstroPhysics Including Relativity, California Institute of Technology, Pasadena, CA 91125, USA \\ ${ }^{3}$ Department of Astronomy, University of Maryland, College Park, MD 20742-2421, USA \\ Received 2011 October 12; accepted 2012 February 6; published 2012 March 29
}

\begin{abstract}
We present numerical relativity results of tidal disruptions of white dwarfs from ultra-close encounters with a spinning, intermediate-mass black hole (IMBH). These encounters require a full general relativistic treatment of gravity. We show that the disruption process and prompt accretion of the debris strongly depend on the magnitude and orientation of the black hole $(\mathrm{BH})$ spin. However, the late-time accretion onto the $\mathrm{BH}$ follows the same decay, $\dot{M} \propto t^{-5 / 3}$, estimated from Newtonian gravity disruption studies. We compute the spectrum of the disk formed from the fallback material using a slim disk model. The disk spectrum peaks in the soft X-rays and sustains Eddington luminosity for 1-3 yr after the disruption. For arbitrary BH spin orientations, the disrupted material is scattered away from the orbital plane by relativistic frame dragging, which often leads to obscuration of the inner fallback disk by the outflowing debris. The disruption events also yield bursts of gravitational radiation with characteristic frequencies of $\sim 3.2 \mathrm{~Hz}$ and strain amplitudes of $\sim 10^{-18}$ for galactic IMBHs. The optimistic rate of considered ultra-close disruptions is consistent with no sources found in the ROSAT all-sky survey. Future missions like Wide-Field X-ray Telescope could observe dozens of events.
\end{abstract}

Key words: accretion, accretion disks - black hole physics - gravitational waves - hydrodynamics - relativistic processes - shock waves - X-rays: bursts

Online-only material: color figures

\section{INTRODUCTION}

Tidal disruptions of stars by black holes (BHs) are fascinating and violent cosmic events, releasing copious amounts of energy in electromagnetic radiation and, in some cases, also accompanied by potentially detectable gravitational wave $(\mathrm{GW})$ emission. The scattered debris from a stellar disruption will yield different radiation patterns depending on the orientation of the orbit. This variety of radiation patterns will provide clues about both the $\mathrm{BH}$ and the internal structure of the disrupted star. As the debris from a tidal disruption showers back into the $\mathrm{BH}$ to form an accretion disk, for instance, it will radiate close to or above the Eddington luminosity for some duration, with the spectrum peaking at UV/X-ray frequencies depending on the BH mass (Rees 1988; Evans \& Kochanek 1989; Cannizzo et al. 1990; Bogdanović et al. 2004; Strubbe \& Quataert 2009). In addition, the unbound debris will quickly scatter over a large volume and may be illuminated by the accretion disk, leading to an optical irradiation spectrum dominated by lines (Bogdanović et al. 2004; Sesana et al. 2008; Strubbe \& Quataert 2009; Clausen \& Eracleous 2011).

When a main-sequence star is disrupted, the super-Eddington outflow is by itself hot enough to be a significant source of optical emission resembling supernova radiation (Kasen \& Ramirez-Ruiz 2009; Strubbe \& Quataert 2009). In these circumstances, instead of outflowing debris, a steady optically thick envelope may reprocess the inner disk radiation (Loeb \& Ulmer 1997; Ulmer et al. 1998). As a consequence, neither the inner disk nor the irradiated debris emission is visible. Another tidal disruption effect arises from the tidal compression perpendicular to the orbital plane. The compression leads

\footnotetext{
4 Hubble Fellow.
}

to the formation of a strong shock that triggers a powerful short outburst (Kobayashi et al. 2004; Guillochon et al. 2009; Rosswog et al. 2009). In some instances, e.g., ultra-close disruptions, the tidal compression could be strong enough to produce a thermonuclear ignition of the star. In such cases, the detonation of the star would be observed as an underluminous supernova (Rosswog et al. 2009).

The variety of proposed radiative signatures often disagree with each other, calling for precise dynamical modeling of tidal disruptions. Early hydrodynamic simulations by Evans \& Kochanek (1989) confirmed a flat distribution of debris mass over its energy range, yielding the predicted $t^{-5 / 3}$ law of fallback accretion rate (Rees 1988). Other numerical investigations addressed the tidal compression and related shock formation; some of these studies used smoothed particle hydrodynamics (SPH) techniques (Kobayashi et al. 2004; Rosswog et al. 2009) and others hydrodynamic grid-based codes (Guillochon et al. 2009). The studies by Rosswog et al. (2009) included nuclear reactions with simplified networks. On the other hand, Bogdanović et al. (2004) looked at the formation of spherical envelopes (Loeb \& Ulmer 1997) using SPH simulations, but the study did not find any conclusive evidence of a spherical structure. At present, tidal disruption simulations that capture all the relevant physics are quite challenging. The simulations require handling, in addition to hydrodynamics, effects from general relativity, nuclear reactions, and magnetic fields. Thus, many important questions are still left unanswered. In particular, there are very few simulations that account for general relativistic effects (Laguna et al. 1993a, 1993b; Kobayashi et al. 2004; Bogdanović et al. 2004). The inclusion of magnetic fields may lead to jet formation.

Very likely, the BHs involved in a tidal disruption will have spins misaligned with the orbital angular momentum of the incoming star. Thus, the disk formed from the tidal debris will 
have angular momentum that is also misaligned with the $\mathrm{BH}$ spin direction (Rees 1988). For thin disks, the Bardeen-Petterson effect will align the $\mathrm{BH}$ spin with the angular momentum of the inner region of the disk (Bardeen \& Petterson 1975), but for the thick disks expected from tidal disruptions, the alignment may not happen (Papaloizou \& Pringle 1983; Fragile et al. 2007; Stone \& Loeb 2012). This misalignment could have important consequences on the emission channels from the disruption, e.g., jet formation. The spin of the BH will also produce distinct effects in an ultra-close encounter when the pericentric radius $R_{\mathrm{p}}$ is comparable to the gravitational radius $R_{\mathrm{g}}=G M_{\mathrm{bh}} / c^{2}$ of the $\mathrm{BH}$. In this strong gravity regime, the details of the disruption will depend not only on the stellar radius, stellar equation of state, and orbital energy but also on the magnitude and orientation of the spin of the BH. In particular, the properties of the accretion will depend on the $\mathrm{BH}$ spin since its magnitude determines the location of the innermost stable circular orbit (ISCO) radius $r_{\text {ISCO }}$, ranging from $r_{\mathrm{ISCO}}=9 R_{\mathrm{g}}$ for a counterrotating maximally spinning $\mathrm{BH}$ to $r_{\mathrm{ISCO}}=1 R_{\mathrm{g}}$ for a corotating maximally spinning $\mathrm{BH}$. The value of spin determines whether the star following the same orbit gets disrupted (Kesden 2011). Furthermore, a misaligned rotating BH will drag around nearby debris (Bardeen et al. 1972; Shapiro $\&$ Teukolsky 1986) and will effectively push this material away from the orbital disruption plane. The net effect will be the formation of a shell-like disruption debris engulfing the $\mathrm{BH}$, as opposed to the traditional S-shape debris observed in Newtonian gravity simulations (Evans \& Kochanek 1989).

The present work aims at exploring the exciting regime of ultra-close encounters (i.e., $R_{\mathrm{p}} \sim$ few $R_{\mathrm{g}}$ ) of a white dwarf (WD) with an intermediate-mass black hole (IMBH). Our main goal is to investigate observational signatures due to strong gravity effects that may shed light on the presence of IMBHs. We consider a carbon-oxygen WD with mass $M_{\mathrm{wd}}=1 M_{\odot}$ and radius (Nauenberg 1972)

$$
\begin{aligned}
R_{\mathrm{wd}} & =1.72 R_{\oplus}\left(\frac{M_{\mathrm{wd}}}{M_{\mathrm{ch}}}\right)^{-1 / 3}\left[1-\left(\frac{M_{\mathrm{wd}}}{M_{\mathrm{ch}}}\right)^{4 / 3}\right]^{3 / 4} \\
& =0.95 R_{\oplus}
\end{aligned}
$$

with $M_{\mathrm{ch}}=1.44 M_{\odot}$ the Chandrasekhar mass and $R_{\oplus}=$ $6371 \mathrm{~km}$ the Earth mean radius. We fix the mass of the IMBH to $M_{\mathrm{bh}}=10^{3} M_{\odot}$. With these choices, $R_{\mathrm{wd}} \simeq 4 R_{\mathrm{g}} \simeq 6000 \mathrm{~km}$. Therefore, the WD and IMBH can be numerically modeled with comparable grid resolutions. The other important scale in the problem is the tidal disruption radius $R_{t}$. For our setup (Rees 1988),

$$
\frac{R_{\mathrm{t}}}{R_{\mathrm{g}}} \simeq 44\left(\frac{R_{\mathrm{wd}}}{0.95 R_{\oplus}}\right)\left(\frac{M_{\mathrm{wd}}}{1 M_{\odot}}\right)^{-1 / 3}\left(\frac{M_{\mathrm{bh}}}{10^{3} M_{\odot}}\right)^{-2 / 3}
$$

gave us ample room to carry out deep penetration encounters.

Currently, there is a large body of evidence for the existence of both solar mass and supermassive $\mathrm{BHs}$ with masses in the range of $10^{5}-10^{8} M_{\odot}$. IMBHs are, however, the missing link between stellar mass and supermassive BHs. Today, only tentative evidence exists (Irwin et al. 2010; Farrell et al. 2009; Davis et al. 2011). On the other hand, WDs are thought to be abundant in spiral galaxies (Evans et al. 1987; Reid 2005) and globular clusters (GCs; Gerssen et al. 2002). Thus, the identification of distinct signals in both GWs and the electromagnetic spectrum (Gould 2011) from a disruption event could potentially guide observations that provide evidence for the existence of IMBHs, as well as insights into the structure of the WD involved.

Our study uses the full machinery of numerical relativity to solve the Einstein equations of general relativity and hydrodynamics. We do not include nuclear reactions. For the ultra-close encounters of our interest, with $R_{\mathrm{p}} \sim$ few $R_{\mathrm{g}}$, a general relativistic description of gravity is needed. However, given the mass ratio $M_{\mathrm{wd}} / M_{\mathrm{bh}} \simeq 10^{-3}$ of the bodies involved and encounters not driven by $\mathrm{GW}$ emission, one does not need to account for dynamical general relativistic gravity. The present study could have been carried out by doing hydrodynamics on the fixed spacetime background provided by the IMBH. However, this would have implied developing a general relativistic hydro code on a fixed background, a code similar to the SPH code developed by one of us to investigate the tidal disruption of main-sequence stars by supermassive BHs (Laguna et al. 1993a, 1993b). We decided instead to take advantage of our numerical relativity code MAYA. The code has demonstrated excellent performance in handling fluid flows in the vicinity of BHs in our studies of binary $\mathrm{BH}$ mergers in astrophysical environments (Bode et al. 2008, 2009, 2012). The other advantage of using the MAYA code is the ability to obtain the GW signal directly from the simulation without having to take recourse to the possibly inaccurate quadrupole formula or the application of post-Newtonian approximations to spacetimes containing a spinning $\mathrm{BH}$.

The paper is organized as follows. In Section 2, we describe how self-consistent general relativistic initial data are constructed. Results from the dynamics of the disruption events are discussed in Section 3. We estimate electromagnetic transient radiation in Section 4 using a slim disk model to compute the spectrum during the fallback phase. We find that the sources shine at Eddington luminosity $\sim 10^{41} \mathrm{erg} \mathrm{s}^{-1}$ for about $1-3 \mathrm{yr}$ and then fade approximately as $\propto t^{-5 / 3}$. We also find that there is a 50/50 chance that the inner disk will be obscured by outflowing debris for a fully misaligned BH spin. In Section 5, we calculate the GW signal produced during the encounters. In Section 6, we estimate the event rates and discuss the observational prospects. We discuss the progress and the limitations of our study in Section 7.

\section{INITIAL DATA AND CODE TESTS}

When using a fully general relativistic code, such as our MAYA code, that is based on a 3+1 formulation of the Einstein equations, the initial data for the dynamical spacetime are composed of the spatial metric $\gamma_{i j}$ and the extrinsic curvature $K_{i j}$ of the initial spacetime hypersurface. All tensor indices are spatial indices and, in this section only, we use units for which $G=c=1$. The metric $\gamma_{i j}$ characterizes the gravitational potentials of the IMBH and WD, and the tensor $K_{i j}$ provides the "velocity" of the metric, or the embedding of the space-like hypersurface, in the spacetime. ${ }^{5}$

Although $\gamma_{i j}$ and $K_{i j}$ are dominated by the IMBH, it is crucial to account for the contributions from the WD in order to correctly include the self-gravity of the WD. The components of $\gamma_{i j}$ and $K_{i j}$ cannot all be freely specifiable. They need to satisfy the following elliptic equations:

$$
\begin{gathered}
8 \Delta \phi+\phi^{-5} \bar{A}_{i j} \bar{A}^{i j}=-16 \pi \phi^{-3} \bar{\rho}_{*}, \\
\partial_{j} \bar{A}^{i j}=8 \pi \bar{J}^{i},
\end{gathered}
$$

\footnotetext{
5 For a review on numerical relativity, we recommend the textbooks by Alcubierre (2008) and Baumgarte \& Shapiro (2010).
} 
where

$$
\begin{aligned}
\gamma_{i j} & =\phi^{4} \delta_{i j} \\
K^{i j} & =\bar{A}^{i j} \phi^{-10} \\
\rho_{*} & =\bar{\rho}_{*} \phi^{-8} \\
J^{i} & =\bar{J}^{i} \phi^{-10} .
\end{aligned}
$$

Above, $\phi$ is a conformal factor, $\delta_{i j}=\operatorname{diag}(1,1,1)$ is the flat spatial metric, and $\Delta$ is its associated Laplace operator. In writing Equations (3) and (4), we have made the customary assumption that the initial physical metric $\gamma_{i j}$ is conformally flat and $K_{i j}$ is trace-free. Equations (3) and (4) are, respectively, the conformal versions of the so-called Hamiltonian and momentum constraints of general relativity (York 1979). The sources $\rho_{*}$ and $J^{i}$ are, respectively, the total energy and momentum densities.

Our approach to solve the Hamiltonian and momentum constraints is to specify the "free" data in Equations (3) and (4) from the solutions to an isolated, spinning $\mathrm{BH}$ and a boosted WD (Löffler et al. 2006).

Let us consider first the solution for a boosted WD. The sources $\bar{\rho}_{*}$ and $\bar{J}^{i}$ in Equations (3) and (4) are obtained from the stress-energy tensor for a perfect fluid (see chapter 5 in Baumgarte \& Shapiro 2010). They read

$$
\begin{gathered}
\bar{\rho}_{*}=\rho\left(1+\varepsilon+\frac{P}{\rho}\right) W^{2}-P, \\
\bar{J}^{i}=\rho\left(1+\varepsilon+\frac{P}{\rho}\right) W^{2} v^{i},
\end{gathered}
$$

where $W=\left(1-\gamma_{i j} v^{i} v^{j}\right)^{-1 / 2}$ is the Lorentz factor and $v^{i}$ is the boost velocity of the WD. In Equations (9) and (10), $\rho$ is the rest-mass density, $P$ is the pressure, and $\varepsilon$ is the specific internal energy density. The values for those quantities are obtained from Tolman-Oppenheimer-Volkoff solutions (Oppenheimer \& Volkoff 1939; Tolman 1939) with a polytropic equation of state $P=K \rho^{\Gamma}$. We use $\Gamma=5 / 3$ appropriate for a non-degenerate gas, which is sufficient to capture the dynamics during the inspiral and disruption phases. During the evolution, we switch to a gamma-law equation of state $P=\rho \varepsilon(\Gamma-1)$. We denote the solutions to Equations (3) and (4) in the absence of the $\mathrm{BH}$ by $\phi_{\mathrm{wd}}$ and $\bar{A}_{i j}^{\mathrm{wd}}$.

Now we consider the solution to the constraints for a spinning $\mathrm{BH}$. In the absence of the WD, $\bar{\rho}_{*}=0$ and $\bar{J}^{i}=0$. In this case, the momentum constraint (4) can be solved analytically (Bowen $\&$ York 1980). The solution reads

$$
\bar{A}_{i j}^{\mathrm{bh}}=\frac{3}{r^{3}}\left(\epsilon_{k i l} S^{l} l^{k} l_{j}+\epsilon_{k j l} S^{l} l^{k} l_{i}\right)
$$

with $l^{i}=x^{i} / r$ and $\epsilon_{i j k}$ the three-dimensional Levi-Civita symbol. Above, $S^{i}$ is the spin vector of the $\mathrm{BH}$.

With the solutions $\bar{A}_{i j}^{\text {wd }}$ and $\bar{A}_{i j}^{\text {bh }}$ at hand, it is clear that the linear superposition $\bar{A}_{i j}=\bar{A}_{i j}^{\text {bh }}+\bar{A}_{i j}^{\text {wd }}$ is the solution to the momentum constraint (4) in the presence of both a WD and a $\mathrm{BH}$. Next, we solve the Hamiltonian constraint (4) with $\bar{A}_{i j}=\bar{A}_{i j}^{\mathrm{bh}}+\bar{A}_{i j}^{\mathrm{wd}}$ and $\bar{\rho}_{*}$ given by Equation (9). We use the popular ansatz

$$
\phi=1+\frac{M_{\mathrm{bh}}}{2 r}+u \text {. }
$$

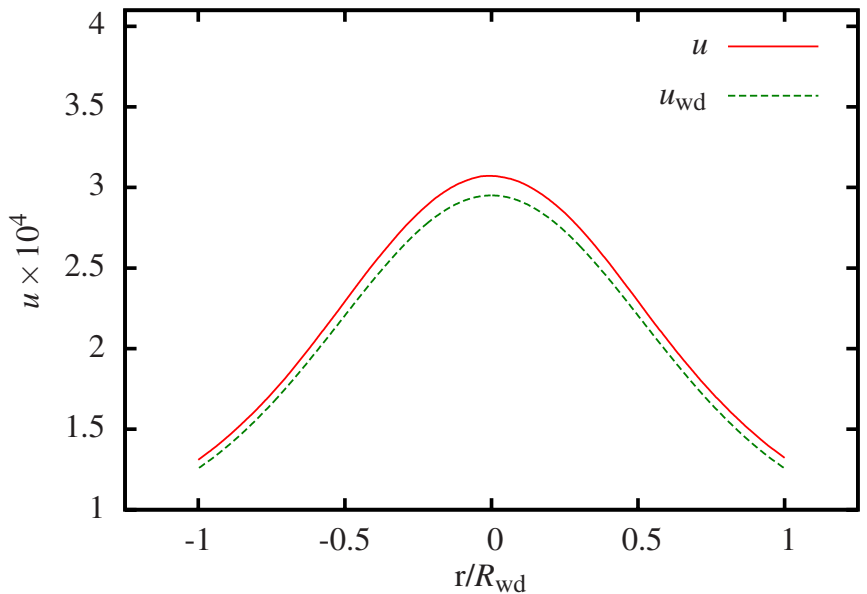

Figure 1. Solution $u$ to Equation (13) for a WD at rest a distance $15 R_{\mathrm{wd}}$ away from a non-spinning IMBH. The solution $u$ provides not only the gravitational potential of the WD but also includes the full nonlinear interactions with the IMBH. For comparison, we also plot $u_{\mathrm{wd}}$, the solution to Equation (13) for a WD in isolation.

(A color version of this figure is available in the online journal.)

Thus, Equation (3) becomes an equation for $u$. In Equation (12), the first term is the flat space solution to Equation (3), the second is the $\mathrm{BH}$ solution, and the third term $u$ includes both the gravitational potential of the WD and the interaction effects with the BH.

To test our initial data method, and in particular to assess the importance of the WD-BH interaction effects, we compute initial_data for a WD initially at rest, that is, $\bar{J}^{i}=0$ and thus $\bar{A}_{i j}^{\mathrm{wd}}=0$. In addition, we assume a non-spinning $\mathrm{BH}$, i.e., $\bar{A}_{i j}^{\text {bh }}=0$. The Hamiltonian constraint (3), with the help of Equation (12), takes the form

$$
\bar{\Delta} u=-16 \pi \phi^{-3} \bar{\rho}_{*} .
$$

The solution to Equation (13) is shown in Figure 1. Also in Figure 1 is the solution $u_{\mathrm{wd}}$ to Equation (13) without the $\mathrm{BH}$, that is, with $M_{\mathrm{bh}}=0$ in Equation (12). The difference between $u$ and $u_{\mathrm{wd}}$ is entirely due to the gravitational interaction of the WD with the BH.

We also tested the ability of our method to produce stable isolated WD models and to conserve rest mass. We found that, over six dynamical times, the central density of the WD did not change more than $1.2 \%$ of its initial value and rest mass was conserved at the $0.1 \%$ level. This is in spite of the adaptive mesh refinement infrastructure we use (CARPET) not being designed to accurately preserve rest-mass conservation when interpolating between different refinement levels.

\section{SIMULATION RESULTS}

\subsection{Tidal Disruption Simulation Parameters}

Our study consists of a series of six simulations. As mentioned before, the IMBH has a mass $M_{\mathrm{bh}}=10^{3} M_{\odot}$, and the WD has a mass $M_{\mathrm{wd}}=1 M_{\odot}$ and radius $R_{\mathrm{wd}} \simeq 6000 \mathrm{~km}$ (Hamada \& Salpeter 1961). The WD mass and radius correspond to a central density $\rho_{\text {initial }}=1.33 \times 10^{7} \mathrm{~g} \mathrm{~cm}^{-3}, \Gamma=5 / 3$, and polytropic constant $K=2.41 \times 10^{-9}\left(\mathrm{~cm}^{3} \mathrm{~g}^{-1}\right)^{\Gamma-1}$. All the simulations start with the WD in a parabolic orbit, in the Newtonian gravity sense, a distance $1.5 R_{\mathrm{t}} \simeq 60 R_{\mathrm{g}}$ along the negative $\hat{x}$-axis away from the IMBH. The orbital angular momentum of the WD is aligned with the $\hat{z}$-axis, as depicted in Figure 2. 


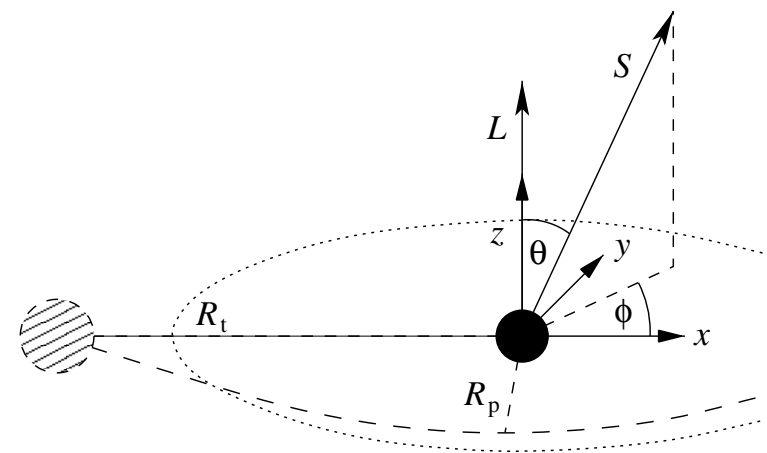

Figure 2. Configuration setup of our simulations. The WD is located at a distance $1.5 R_{\mathrm{t}} \simeq 60 R_{\mathrm{g}}$ along the negative $\hat{x}$-axis away from the $\mathrm{BH}$. The orbital angular momentum $\vec{L}$ of the WD is aligned with the $\hat{z}$-axis. The orientation of the $\mathrm{BH}$ spin $\vec{S}$ is determined by the standard $\theta$ and $\phi$ angles in a right-handed spherical coordinate system.

Table 1

Simulation Parameters

\begin{tabular}{lllcc}
\hline \hline Run & $\beta$ & $a^{\star}$ & $\theta$ & $\phi$ \\
\hline B6S0 & 6 & 0.0 & $0^{\circ}$ & $0^{\circ}$ \\
B6Su & 6 & 0.6 & $0^{\circ}$ & $0^{\circ}$ \\
B6Sd & 6 & 0.6 & $180^{\circ}$ & $0^{\circ}$ \\
B6Si & 6 & 0.6 & $90^{\circ}$ & $0^{\circ}$ \\
B6Sa & 6 & 0.6 & $63^{\circ}$ & $90^{\circ}$ \\
B8Sa & 8 & 0.6 & $63^{\circ}$ & $90^{\circ}$ \\
\hline
\end{tabular}

Note. $\beta=R_{\mathrm{t}} / R_{\mathrm{p}}$ is the penetration factor, $a^{\star}=|\vec{S}| / M^{2}$ is the dimensionless spin parameter of the central black hole, $\theta$ and $\phi$ are the angles between $\vec{S}$ and the orbital angular momentum ( $\hat{z}$-axis) and the radial direction joining the IMBH and the WD ( $\hat{x}$-axis), respectively.

Table 1 lists the configurations used in the simulations where $\beta=R_{\mathrm{t}} / R_{\mathrm{p}}$ is the penetration factor for an orbit with pericentric distance $R_{\mathrm{p}}$, and $a^{\star}=|\vec{S}| / M^{2}$ is the magnitude of the dimensionless spin parameter of the IMBH. The angles $\theta$ and $\phi$ determine the direction of the BH spin. They are the standard angles in a right-handed spherical coordinate system as depicted in Figure 2. We use the following convention to label the cases we studied. If a case is labeled "BxSy," the WD had penetration factor $\beta=x$. In addition, the value of $y$ denotes a non-spinning $\mathrm{BH}(y=0)$ or the BH's spin orientation: (up, down, in-plane, arbitrary) for $y=(u, d, i, a)$, respectively.

In all the simulations, we employ eight levels of mesh refinement with radii $1.24 R_{\mathrm{g}} \times 2^{\ell}(0 \leqslant \ell \leqslant 7)$ centered at the $\mathrm{BH}$ location. In addition to these refinement levels, we surround the WD during the pre-disruption phase with five additional nested boxes of radii $4.96 R_{\mathrm{g}} \times 2^{\ell}(0 \leqslant \ell \leqslant 4)$. The resolution on the finest refinement is $R_{\mathrm{g}} / 19.35$, and the resolution on the level covering the WD is $R_{\mathrm{g}} / 9.675$. This mesh refinement setup becomes insufficient when the WD begins to be disrupted. At this point, we turn off the boxes tracking the WD and construct a larger mesh with resolution $R_{\mathrm{g}} / 9.675$ that includes both the IMBH and the highly distorted WD. Once the expanding debris has cleared the inner region and the density has dropped, we turn off this level again to speed up the simulation.

The mesh refinements affect our ability to conserve rest mass throughout the computational domain, in particular if the meshes are moving, created, or removed. Figure 3 displays the relative gain/loss of rest mass in the computational domain, $\delta M / M_{\mathrm{wd}}=\left(M-M_{\mathrm{wd}}\right) / M_{\mathrm{wd}}$, where $M$ is the total relativistic rest mass and $M_{\mathrm{wd}}$ is the initial WD rest mass. The computed

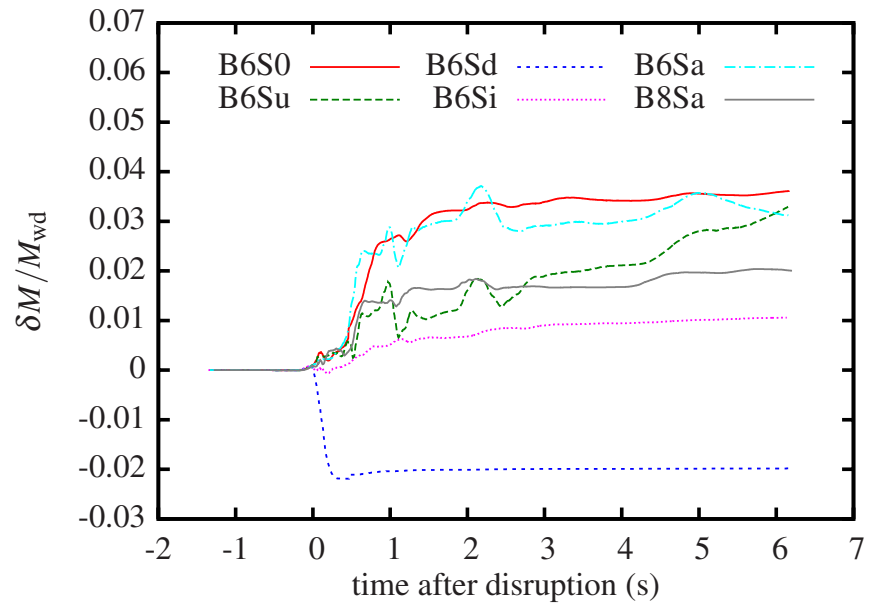

Figure 3. Relative gain/loss of rest mass in the computational domain, $\delta M / M_{\mathrm{wd}}=\left(M-M_{\mathrm{wd}}\right) / M_{\mathrm{wd}}$, where $M$ is the total relativistic rest mass and $M_{\mathrm{wd}}$ is the initial WD rest mass. The total relativistic rest mass $M$ takes into account fluxes into the $\mathrm{BH}$. The only simulation run displaying a net mass loss is the case B6Sd, in which almost all the material accretes promptly. In the other cases, most of the mass gain happens in the short high-compression phase when the WD is at its periapsis point close to the $\mathrm{BH}$.

(A color version of this figure is available in the online journal.)

value of $M$ takes into account the mass loss through the $\mathrm{BH}$ horizon and the outer boundary of the computational domain. We find tolerable mass conservation, with violations of less than $4 \%$ over the course of the simulations. Runs B6Su and B6S0 display the worst mass conservation. As we shall see, these are the cases in which most of the material of the star ( $\gtrsim 95 \%)$ escapes the $\mathrm{BH}$, forming an expanding cloud that reaches the coarsest mesh refinements. Note also that most of the errors in mass conservation accumulate after the disruption. Run B6Sd is the only run that loses mass. This is the case in which the IMBH accretes almost all of the star during the initial passage of the WD.

\subsection{Tidal Compression}

The first stage in a tidal disruption encounter consists of the deformations that the incoming star experiences due to the tidal forces. In the orbital plane of the star, there are two competing tidal deformations. One of them is tidal stretching along the radial direction joining the IMBH with the WD. The other is tidal compression perpendicular to this radial direction. In the direction perpendicular to the orbital plane, there is also tidal compression. This compression, or squeezing, is stronger than that in the orbital plane because in the latter the radial direction changes rapidly as the star reaches periapsis, thus modifying the "contact point" in the star of the in-plane compression. We concentrate here on the compression in the direction perpendicular to the orbital plane. This compression could, in principle, generate enough heat to detonate the star (Luminet 1985; Luminet \& Marck 1985; Luminet \& Pichon 1989) by igniting nuclear reactions at the core of the WD (Rosswog et al. 2009). To investigate whether a nuclear ignition is likely to happen from tidal compression, we plot in Figure 4 the instantaneous maximum temperature of the star as a function of the density at that location. To a good approximation, this density is also the instantaneous maximum density. The instantaneous maximum temperature is computed as the massdensity-weighted temperature average of the portion of the star whose numerical cells have the largest internal energy and make up for $10 \%$ of its mass. 


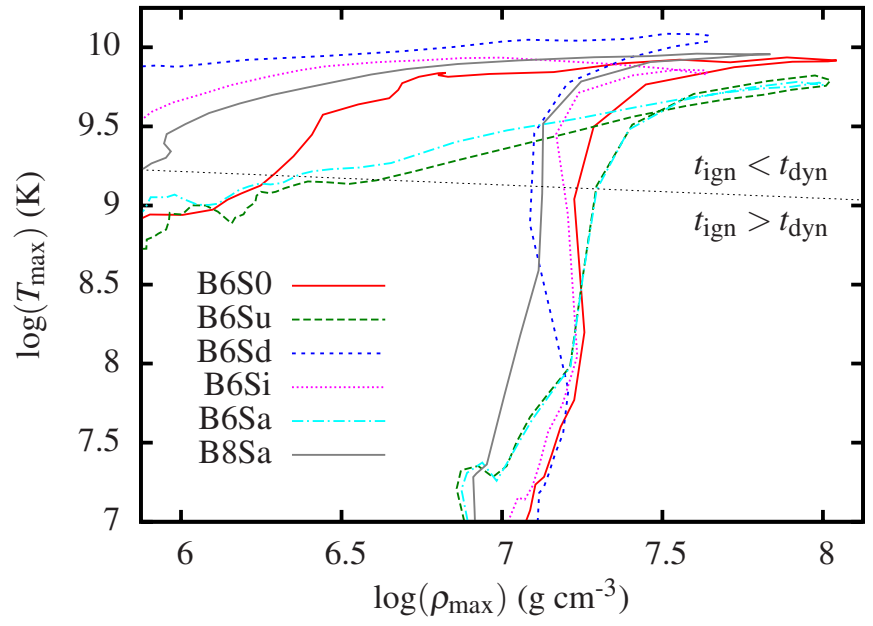

Figure 4. Instantaneous maximum temperature as a function of the density at the same location. We compute the density-weighted temperature average of the hottest cells in the grid, which make up $10 \%$ of the total rest mass of the WD. The dotted black line separates regions in parameter space where the ignition time as estimated from Figure 2 of Dursi \& Timmes (2006) is larger/smaller than the dynamical timescale of the WD, i.e., the region of successful explosion from the region where the explosion fails. Densities smaller than $10^{7} \mathrm{~g} \mathrm{~cm}^{-3}$ are not present in Figure 2, and the estimates for the ignition time in this region are based on simple linear extrapolation. In all cases investigated, the ignition timescale $t_{\text {ign }}$ of the WD is much shorter than the dynamical timescale $t_{\text {dyn }}=1 / \sqrt{G \rho} \gtrsim 0.38 \mathrm{~s}$ of the WD.

(A color version of this figure is available in the online journal.)

We calculate temperatures $T$ from the thermal specific energy as (see Lee et al. 2005)

$$
m_{p} n_{\mathrm{nuc}} \varepsilon_{\mathrm{th}}=\frac{3}{2} k_{B} T\left(N_{\mathrm{ion}}+N_{e}\right) n_{\mathrm{nuc}}+a_{\mathrm{BB}} T^{4},
$$

with

$$
\varepsilon_{\text {th }}=\varepsilon-\frac{K_{\text {initial }}}{\Gamma-1} \rho^{\Gamma-1},
$$

where the second term in Equation (15) is the "cold" specific internal energy in the absence of shocks. $N_{e}$ is the number of electrons per nucleon making up the gas, $N_{\text {ion }}$ is the number of ions per nucleon, $n_{\text {nuc }}$ is the number density of nucleons in the gas, $m_{p}$ is the proton (nucleon) mass, $k_{B}$ is the Boltzmann constant, and $a_{\mathrm{BB}}$ here is the radiation constant. For simplicity, we assume $N_{\text {ion }}=1 / 14$ for an equal mixture of oxygen and carbon atoms. For $N_{e}$, which depends on the ionization state of the plasma, we use $N_{e}=0$ for $T \lesssim 5 \times 10^{6} \mathrm{~K}$ and $N_{e}=1 / 2$ for $5 \times 10^{6} \mathrm{~K} \lesssim T \lesssim 1 \times 10^{10} \mathrm{~K}$. That is, we ignore any partial ionization states of the constituent atoms at low temperatures.

In all cases investigated, the maximum temperature and density displayed in the tracks of Figure 4 reach high enough values to trigger nuclear burning in the star (cf. Figure 2 of Dursi \& Timmes 2006). Interestingly, the temperature-density tracks in Figure 4 are qualitatively similar to those by Rosswog et al. (2009), who included nuclear networks.

In Table 2, we list the maximum temperature $T_{\max }$ and compression $\rho_{\max } / \rho_{\text {initial }}$. Here, $T_{\max }$ and $\rho_{\max }$ are the maximum temperature and density attained over the course of the evolution, respectively. We also list the "actual" penetration factor $\beta^{*}=R_{\mathrm{t}} / R_{\mathrm{p}}^{*}$. Recall that the orbital parameters, and in particular $R_{\mathrm{p}}$, used to set the WD in a parabolic orbit were obtained using Newtonian gravity. Because of relativistic effects, the value of $R_{\mathrm{p}}^{*}$ will differ from the Newtonian estimate $R_{\mathrm{p}}$, and thus

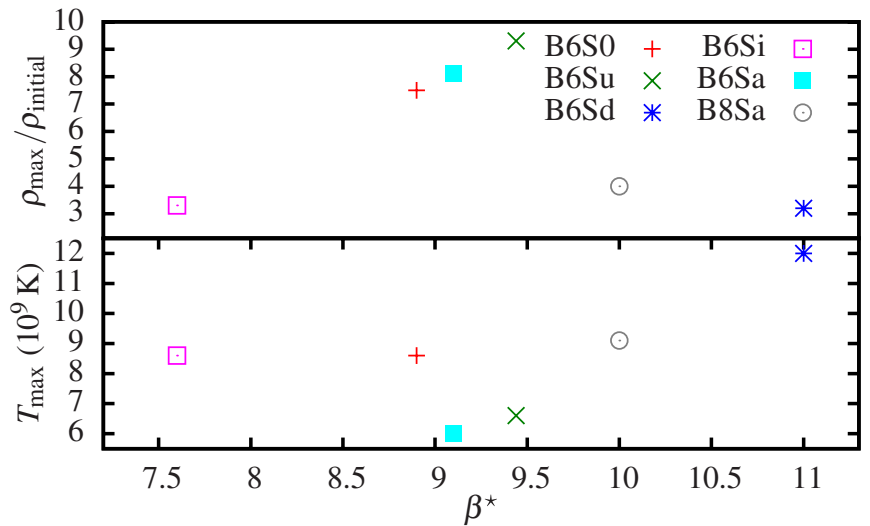

Figure 5. Maximum density $\rho_{\max }$ (top) and temperature $T_{\max }$ as a function of $\beta^{*}$ for the values in Table 2 .

(A color version of this figure is available in the online journal.)

Table 2

Actual Penetration Factor $\beta^{*}$ Measured from the Simulations

\begin{tabular}{llccrr}
\hline \hline Run & $\beta^{*}$ & $\begin{array}{c}T_{\max } \\
\left(10^{9} \mathrm{~K}\right)\end{array}$ & $\rho_{\max } / \rho_{\text {initial }}$ & \multicolumn{1}{c}{$f_{\text {acc }}$} & \multicolumn{1}{c}{$f_{\text {unb }}$} \\
\hline B6S0 & 8.9 & 8.6 & 7.5 & $68 \%$ & $19 \%$ \\
B6Su & 9.44 & 6.6 & 9.3 & $<1 \%$ & $60 \%$ \\
B6Sd & 11 & 12 & 3.2 & $>99 \%$ & $<0.5 \%$ \\
B6Si & 7.6 & 8.6 & 3.3 & $65 \%$ & $22 \%$ \\
B6Sa & 9.1 & 6.0 & 8.1 & $2 \%$ & $67 \%$ \\
B8Sa & 10 & 9.1 & 4.0 & $43 \%$ & $34 \%$ \\
\hline
\end{tabular}

Notes. Maximum temperature $T_{\max }$ and compression $\rho / \rho_{\text {initial }}$ with $\rho_{\text {initial }}=$ $1.33 \times 10^{7} \mathrm{~g} \mathrm{~cm}^{-3}$. Fraction $f_{\text {acc }}$ of the star accreted during the first $2 \mathrm{~s}$ after disruption; unbound fraction $f_{\text {unb }}$ at the end of the simulation at $\sim 6 \mathrm{~s}$.

$\beta \neq \beta^{*}$. In our simulations, we define $R_{\mathrm{p}}^{*}$ as the distance of closest approach of the point within the WD where $T_{\max }$, or equivalently $\rho_{\max }$, is found. Note that for the B6Su and B6Sd cases, $T_{\max }$ almost doubles and $\rho_{\max }$ triples when going from a spin aligned with the orbital angular momentum to one that is anti-aligned. This is because in the anti-aligned case (B6Sd), the WD penetrates closer, $\beta^{*}=11$, than in the aligned case $(\mathrm{B} 6 \mathrm{Sd}), \beta^{*}=9.4$. Figure 5 shows the maximum temperature $T_{\max }$ and maximum density $\rho_{\max }$ as a function of the actual penetration factor $\beta^{*}$ from Table 2 . Because of the small range of values covered by $\beta^{*}$, it is not possible to verify the scaling $T_{\max } \propto \beta^{2}$ and $\rho_{\max } \propto \beta^{3}$ suggested by Carter \& Luminet (1982).

\subsection{Outflowing Debris and Spin}

All cases showed that the mass and orientation of the debris after disruption are strongly affected by the BH spin. This is because the spin determines the location of ISCO radius, and it is also responsible for frame-dragging effects. In particular, frame dragging pulls the material out of the orbital plane, thereby changing the orientation of the resulting disk.

We first discuss three disruptions: disruption by a nonspinning IMBH (case B6S0), by an IMBH with spin aligned (case B6Su), and by an IMBH with spin anti-aligned (case B6Sd) relative to the orbital angular momentum (see Table 2). For the spinning BH cases, the magnitude of the dimensionless spin parameter $a^{*}$ was kept at 0.6. With this spin magnitude, $r_{\mathrm{ISCO}}=5670 \mathrm{~km}$ for the case B6Su, and $r_{\mathrm{ISCO}}=11,600 \mathrm{~km}$ for the case B6Sd. In the non-spinning case, $r_{\text {ISCO }}=9000 \mathrm{~km}$ (McClintock et al. 2011). All three cases had penetration factors 


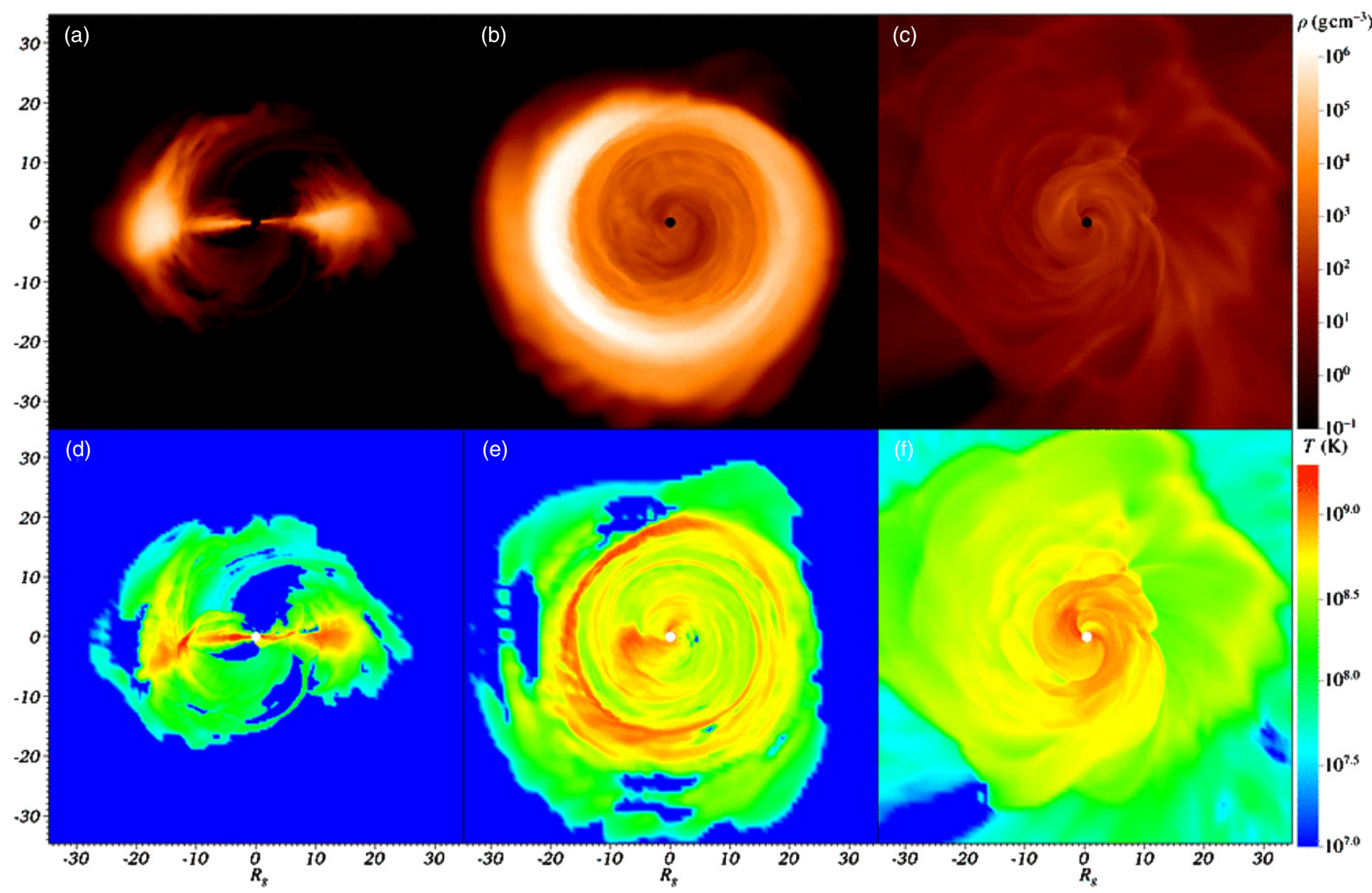

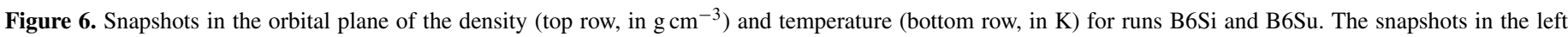
column are for the case $\mathrm{B} 6 \mathrm{Si} \sim 0.5 \mathrm{~s}$ after disruption. The middle and right columns correspond to case B $6 \mathrm{Su} \sim 0.5 \mathrm{~s}$ and $\sim 5.9 \mathrm{~s}$ after disruption, respectively.

(A color version of this figure is available in the online journal.)

$\beta=R_{\mathrm{t}} / R_{\mathrm{p}}=6$. From Equation (2), $R_{\mathrm{t}}=40 R_{\mathrm{g}}=60,000 \mathrm{~km}$; thus, a penetration factor of 6 translates to a pericentric distance $R_{\mathrm{p}} \simeq 10,000 \mathrm{~km}$. We then expect that the WD in the retrograde case B6Sd will pass within the ISCO, in the prograde case B6Su the star will mostly stay outside of the ISCO, and in the case B6S0 the WD will graze by the ISCO. Because $r_{\text {ISCO }}>R_{\mathrm{p}}$ in the B6Sd case, almost all of the star is accreted by the BH soon after the disruption. For the cases B6S0 and B6Su, on the other hand, most of the stellar debris escapes direct capture, expanding away from the hole. The debris cloud has a shape resembling a thick circular arch. This arch-like cloud eventually closes up and forms a disk around the $\mathrm{BH}$.

In all cases, the leading edge of the tidally disrupted star wraps around the $\mathrm{BH}$ and crashes into the trailing material, creating a hot region. For the B6Su, B6Sd, and B6S0 cases, this hot region is mostly contained within the equatorial plane and moves outward from the central region in the form of a spiraling disturbance in the debris disk, eventually appearing on the debris surface. Figure 6 shows snapshots in the orbital plane of the density (top row) and temperature (bottom row) for runs B6Si and B6Su. The snapshots in the left column are for the case B6Si $\sim 0.5 \mathrm{~s}$ after disruption. The middle and right columns correspond to case $\mathrm{B} 6 \mathrm{Su} \sim 0.5 \mathrm{~s}$ and $\sim 5.9 \mathrm{~s}$ after disruption, respectively. In the aligned case $\mathrm{B} 6 \mathrm{Su}$, the hot region forms a spiral that moves outward from the point where the leading edge of the WD intersected with the tail material. At late times this feature has been washed out. In run B6Si the hot region is much smaller; frame dragging by the $\mathrm{BH}$ has pulled material out of the plane into a shell surrounding the $\mathrm{BH}$.
For the cases in which the spin of the $\mathrm{BH}$ is not aligned with the orbital angular momentum of the binary (i.e., B6Si, $\mathrm{B} 6 \mathrm{Sa}$, and B8Sa cases), frame dragging is effectively able to wrap the debris material around the $\mathrm{BH}$. This effect-which is not captured by Newtonian calculations-greatly changes the geometry of the debris, blanketing the central region with a shell-like cloud of gas. We will address the observational consequences of the material surrounding the $\mathrm{BH}$ in the next section. The effect of the misalignment is evident from Figure 6 in the remarkable differences of the spiral patterns observed between the left and middle snapshots at $\sim 0.5 \mathrm{~s}$ after disruption. The left panels are for the B6Si case, in which the BH spin direction is perpendicular to the orbital angular momentum. On the other hand, the middle panels are for the B6Su case, in which the BH spin is aligned with orbital angular momentum. The observed differences are a consequence of how the debris responds to the orientation of the $\mathrm{BH}$ spin.

\subsection{Prompt and Late Accretion}

We observe an initial prompt phase of accretion when the star swings by the $\mathrm{BH}$. This prompt accretion phase is followed by an intermediate phase in which material initially deflected by the $\mathrm{BH}$ slows down and begins to accrete. At late times, long after the end of our simulations, the characteristic $t^{-5 / 3}$ fallback behavior takes over.

Figure 7 shows the flow of matter through the IMBH's horizon during the first $6 \mathrm{~s}$ after the disruption takes place. The instant labeled $t_{\text {tidal }}$ is the time at which the star enters the tidal radius. The horizontal dashed line labeled "noise" denotes the level 


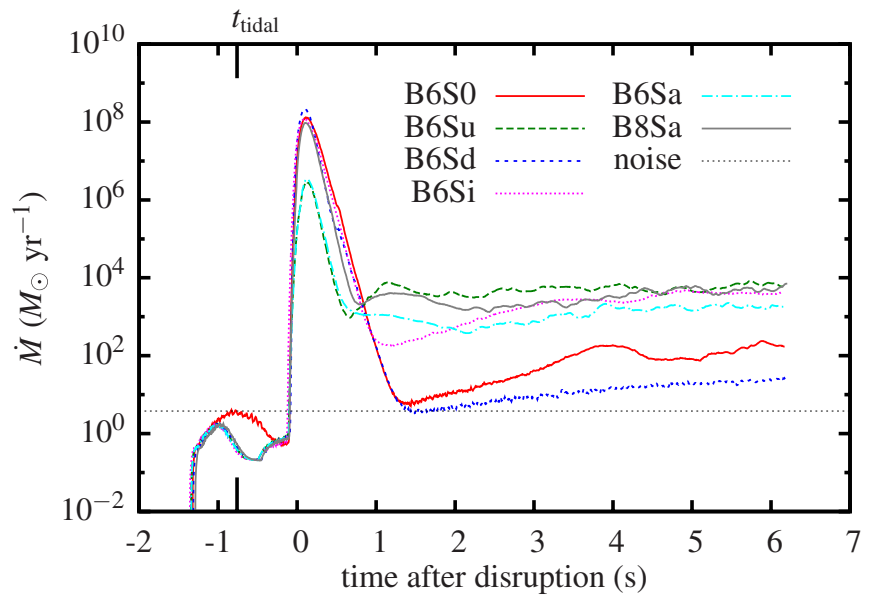

Figure 7. Accretion rate during the simulation. $t_{\text {tidal }}$ indicates the approximate time when the WD enters within the tidal radius $R_{\mathrm{t}}$. The horizontal dotted black line indicates the mass accretion that we observe initially around the black hole. It is a purely numerical artifact from the atmosphere used to model the vacuum regions.

(A color version of this figure is available in the online journal.)

of accretion due to the atmosphere used to model the vacuum regions. We observe that the magnitude of the prompt accretion rate decreases the more prograde an orbit is. Both $\mathrm{B} 6 \mathrm{~S} 0$ and $\mathrm{B} 6 \mathrm{Su}$ disrupt at comparable distances from the central $\mathrm{BH}$, but the peak accretion rate differs by almost two orders of magnitude.

As already pointed out in Section 3.3, the BH spin strongly affects what fraction of the star is accreted onto the $\mathrm{BH}$ soon ( $\sim 2 \mathrm{~s})$ after the star disrupts. Table 2 shows this accreted fraction for each case. In the B6Sd case, the star is completely accreted. On the other hand, the debris in the B6Su and B6Sa cases escapes almost in its entirety during the flyby. Notice also from Table 2 that the prompt accretion is also correlated with the maximum density $\rho_{\max }$ the WD is able to reach as a consequence of the tidal compression.

The late-time behavior consists of material raining back onto the $\mathrm{BH}$ with a characteristic rate of accretion $\dot{M} \propto$ $t^{-5 / 3}$ (Rees 1988; Phinney 1989). This rate is maintained as long as $d M / d \varepsilon_{\text {kin }}$, with $\varepsilon_{\text {kin }}$ being the specific kinetic energy, is approximately constant (Lodato et al. 2009). We follow Rosswog et al. (2009) and compute the fallback time for each fluid element at a time long after disruption, based on data available at the end of the simulation. At this time, hydrodynamical interactions between the fluid elements are small, and each fluid element moves on an almost geodesic orbit. Figure 8 shows the results of these calculations. Clearly, we recover the expected $t^{-5 / 3}$ behavior for return times longer than $t \gtrsim 200 \mathrm{~s}$. Note that earliest times in Figure 8 are comparable to the late-time accretion rates found during the simulation in Figure 7.

\section{ELECTROMAGNETIC SIGNATURES}

Ultra-close (i.e., $R_{\mathrm{p}} \sim$ few $R_{\mathrm{g}}$ ) tidal disruptions are violent events. The conversion of even a small fraction of gravitational energy into light would result in a powerful electromagnetic signature. In this section, we will use the hydrodynamics results from our simulations to make order-of-magnitude estimates of the tidal disruption flare's luminosity, its characteristic photon energy, and the fallback material's electromagnetic signature as it eventually forms a slim accretion disk near the $\mathrm{BH}$.

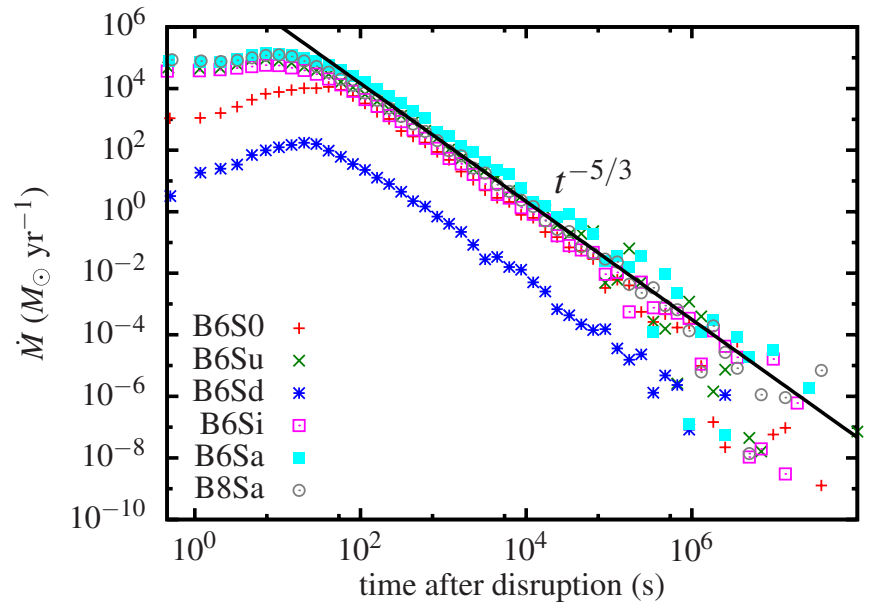

Figure 8. Mass accretion rates computed from the estimated fallback times of the fluid elements. The solid line represents the $t^{-5 / 3}$ falloff predicted in Rees (1988) and Phinney (1989).

(A color version of this figure is available in the online journal.)

When the WD descends into the potential well of the $\mathrm{BH}$, with the typical dynamical timescale of $\sim 1 \mathrm{~s}$, it acquires energy in excess of $\sim 10^{53} \mathrm{erg}$. Even if a tiny fraction of this energy gets converted into radiation on this dynamical timescale, it would lead to a huge luminosity that would be noticeable at cosmological distances. Furthermore, since the WD undergoes a substantial acceleration in the deep gravitational potential of the $\mathrm{BH}$, the streams of matter from the disruption will approach speeds $V_{\mathrm{p}} \simeq\left(G M_{\mathrm{h}} / R_{\mathrm{p}}\right)^{1 / 2} \simeq c\left(\beta R_{\mathrm{g}} / R_{\mathrm{t}}\right)^{1 / 2} \simeq 0.4 \beta_{6} c$, comparable to the speed of light. The streams of tidal debris later collide and transform a portion of their kinetic energy into thermal energy. Being heated by this process in excess of $10^{9} \mathrm{~K}$, the dense hot plasma quickly produces photons (Krolik \& Piran 2011). These photons, however, are effectively trapped by the debris, capping the luminosity around the Eddington luminosity (Eddington 1926)

$$
L_{\mathrm{Edd}}=\frac{4 \pi c G M_{\mathrm{h}} m_{p}}{\sigma_{T}}=1.4 \times 10^{41}\left(\frac{M_{\mathrm{h}}}{10^{3} M_{\odot}}\right) \mathrm{erg} \mathrm{s}^{-1},
$$

where $m_{p}$ is the proton mass and $\sigma_{T}$ is the Thomson crosssection. Note that the equilibrium between the gravitational and spherical radiation fields is reached at a luminosity $L=$ $2 L_{\text {Edd }}=2.8 \times 10^{41} \mathrm{erg} \mathrm{s}^{-1}$ due to composition effects. The system can by far exceed $L_{E d d}$ in the presence of a collimated outflow, especially in the first several minutes after the disruption. We elaborate on this tidal disruption phenomenon in a subsequent paper (R. V. Shcherbakov et al. 2012, in preparation). In the present manuscript we limit ourselves to electromagnetic signatures at late times of months to years.

When the accretion rate falls below the Eddington rate $\dot{M}_{\text {Edd }}$, the accretion becomes radiatively efficient, radiating mostly in X-rays with luminosity $\sim L_{\text {Edd }}$. Assuming an efficiency of $\epsilon=0.1$, this luminosity translates into an accretion rate

$$
\dot{M}_{\mathrm{Edd}}=\frac{L_{\mathrm{Edd}}}{0.1 c^{2}}=2.3 \times 10^{-5}\left(\frac{M_{\mathrm{h}}}{10^{3} M_{\odot}}\right) M_{\odot} \mathrm{yr}^{-1} .
$$

The time elapsed until the accretion rate drops to $\dot{M}_{\text {Edd }}$ can be estimated based on the fallback times reported in Section 3. We have demonstrated that the famous $t^{-5 / 3}$ fallback law (Rees 1988) is also present in our ultra-close encounters. From 
Figure 8 , we observe that the accretion rate in all but the B6Sd case drops below $\dot{M}_{\text {Edd }}$ at times of $t_{\text {Edd }} \sim 1-2$ yr. Thus, the tidal events we are considering can, in principle, shine at Eddington luminosities for many years. The actual luminosity could be substantially sub-Eddington, though, if outflowing debris produced while the $\mathrm{BH}$ swallows matter at super-Eddington rates obscures the accretion flow. In this section, we will first estimate the disk emission within a slim disk model and then consider the added effects of obscuration by the outflowing debris.

\subsection{Slim Disk Model}

For our study we employ a slim disk model, developed and summarized in Abramowicz et al. (1988), Lasota (1994), Kawaguchi (2003), and Sadowski (2009), of a simple one-zone vertically integrated dynamical model. We compute modified blackbody bremsstrahlung emission from the disk. For our radiation estimates, we assume that the angular momentum of the marginally bound debris accreting at late times, and thus the angular momentum of the disk, is aligned with the orbital angular momentum of the incoming WD. The disk model will then, in principle, be misaligned with the spin of the $\mathrm{BH}$. We presume that, despite the misalignment, the slim disk extends down to ISCO radius computed for the aligned case. In our situation it is challenging to find a more reasonable set of assumptions for a geometrically thick slim disk. The ultimate answers require performing full numerical relativity simulations of the fallback regime, a task requiring modeling dynamical timescales $10^{6}$ times longer than those addressed in the present study.

We take our heavy WD to consist of approximately $50 \%$ carbon and $50 \%$ oxygen as suggested by stellar evolution computations by Mazzitelli \& Dantona (1986). The same heavy WD composition was assumed by both Rosswog et al. (2009) and Krolik \& Piran (2011). Thus, there are 1/14 nuclei and 1/2 electrons per one nucleon with mass $\simeq m_{p}$. In terms of nucleon density, the ion and electron densities are

$$
n_{\mathrm{ion}}=\frac{1}{14} n_{\mathrm{nuc}} \quad \text { and } \quad n_{e}=\frac{1}{2} n_{\mathrm{nuc}},
$$

respectively. The corresponding non-relativistic heat capacities of neutral and fully ionized gas per single nucleon of temperature $T$ are

$$
c_{\text {neutral }}=\frac{3}{28} k_{B} T \quad \text { and } \quad c_{\text {ion }}=\frac{6}{7} k_{B} T,
$$

respectively. The energy of full ionization is $E_{\mathrm{CNO}}=$ $\left\langle Z^{2}\right\rangle 13.6 \mathrm{eV}=680 \mathrm{eV}$ for the assumed composition, where $Z \approx 7$ is the charge of the nucleus. A CNO WD is thus fully ionized at $T>T_{\text {ion }} \sim 5 \times 10^{6} \mathrm{~K}$. The molecular mass of the fully ionized plasma is $\mu=1.75$, and its density is $\rho=m_{p} n_{\text {nuc }}$.

A fully self-consistent model of a slim disk requires an estimate of the disk scale height $H$. For a disk in the super-Eddington regime, $H / r=1$ (Abramowicz et al. 1988; Kawaguchi 2003; Strubbe \& Quataert 2009). In our simulations, circularization of debris happens within a distance $30 R_{\mathrm{g}}$ from the $\mathrm{BH}$. We therefore consider a truncated disk located between the ISCO radius $r_{\text {ISCO }}$ and the maximum radius $r_{\max }=30 R_{\mathrm{g}}$. The viscosity parameter is taken to be $\alpha=0.1$. We solve the equations for the central temperature as detailed below, taking into account the effects of viscous heating, emission, and the finite heat capacity of matter. For simplicity, we follow Shakura \& Sunyaev (1973) and set the viscous stress at the ISCO to zero. The energy release per unit disk area is then

$$
F_{+}=\frac{3 r_{\mathrm{g}} \dot{M} c^{2}}{8 \pi r^{3}}\left[1-\left(\frac{r_{\mathrm{ISCO}}}{r}\right)^{1 / 2}\right] .
$$

The accretion rate is expressed as

$$
\dot{M}=4 \pi r H \rho_{c} v_{r}
$$

where $\rho_{c}$ is the one-zone density and $v_{r}>0$ is the inflow velocity.

We adopt a beta disk model where the viscous stress is given by $t_{r \phi}=\alpha p_{\mathrm{g}}$, with $p_{\mathrm{g}}$ being the gas pressure (Sakimoto $\&$ Coroniti 1981). A beta disk model is one of the three options. In the standard Shakura \& Sunyaev (1973) model, $t_{\phi} \propto p_{\text {tot }}=p_{\mathrm{g}}+p_{\text {rad }}$, whereas in the "mean field" models, $t_{\phi} \propto \sqrt{p_{\mathrm{g}} p_{\text {tot }}}$ (see, e.g., Done \& Davis 2008 for a review). There is no definitive answer yet about which model better describes stress in a radiation-dominated accretion disk. The beta disk model $t_{r \phi}=\alpha p_{\mathrm{g}}$ assumption leads to a radial velocity of plasma

$$
v_{r} \approx \alpha c_{s}=\alpha \sqrt{\frac{k_{B} T_{c}}{\mu m_{p}}},
$$

where $c_{s}$ is the isothermal sound velocity. The expression (22) for radial velocity, though not fully self-consistent, helps to avoid the infinite increase of the density near the ISCO inherent to early slim disk models (Abramowicz et al. 1988).

The accretion disk emits predominantly bremsstrahlung radiation. Since the scattering cross-section in the disk is much higher than the absorption cross-section, the emission spectrum is described by a modified blackbody spectrum (Rybicki \& Lightman 1979; Czerny \& Elvis 1987; Kawaguchi 2003). The photon production rate via bremsstrahlung is (Rybicki \& Lightman 1979; Katz et al. 2010)

$$
Q=\sqrt{\frac{8}{\pi}} \alpha_{f} Z^{2} n_{\mathrm{ion}} n_{e} \sigma_{T} c \sqrt{\frac{m_{e} c^{2}}{k_{B} T_{e}}} \Lambda_{\mathrm{eff}} g_{\mathrm{eff}} \mathrm{cm}^{-3} \mathrm{~s}^{-1},
$$

where $\Lambda_{\text {eff }} \sim g_{\text {eff }} \sim 1, \quad \alpha_{f} \approx 0.00729$ is the fine structure constant, and $\sigma_{T}$ is the Thomson cross-section. With the high densities, the production rate $Q$ is high so the radiation quickly dominates internal energy and pressure.

Let us estimate the equilibrium temperature of this radiationdominated flow. The typical nucleon density in the simulation varies from $n_{\text {nuc }} \sim 10^{25} \mathrm{~cm}^{-3}$ near the $\mathrm{BH}$ to a minimum of $n_{\text {min, nuc }} \sim 8 \times 10^{20} \mathrm{~cm}^{-3}$. The typical virial temperature is $T_{\text {ion }} \sim 10^{11} \mathrm{~K}$. As photons are produced, the equilibrium implies

$$
\frac{6}{7} n_{\text {nuc }} k_{B} T_{\text {ion }}=a_{\mathrm{BB}} T_{\mathrm{ph}}^{4},
$$

leading to photon temperatures $T_{\mathrm{ph}} \sim 3 \times 10^{8} \mathrm{~K}$. The timescale for reaching equilibrium can be estimated as

$$
t_{\mathrm{eq}} \sim \frac{a_{\mathrm{BB}} T_{\mathrm{ph}}^{4}}{k_{B} T_{\mathrm{ph}}} \frac{1}{Q\left(T_{\mathrm{ph}}\right)}=9 \times 10^{10} \frac{T_{\mathrm{ion}}^{7 / 8}}{n_{\text {nuc }}^{9 / 8}} \sim 10^{-8} \mathrm{~s},
$$

which is significantly shorter than the dynamical timescale $t_{\text {orb }} \sim 1 \mathrm{~s}$.

We work in the diffusion approximation of high optical depth to find the surface temperature. The corresponding scattering 


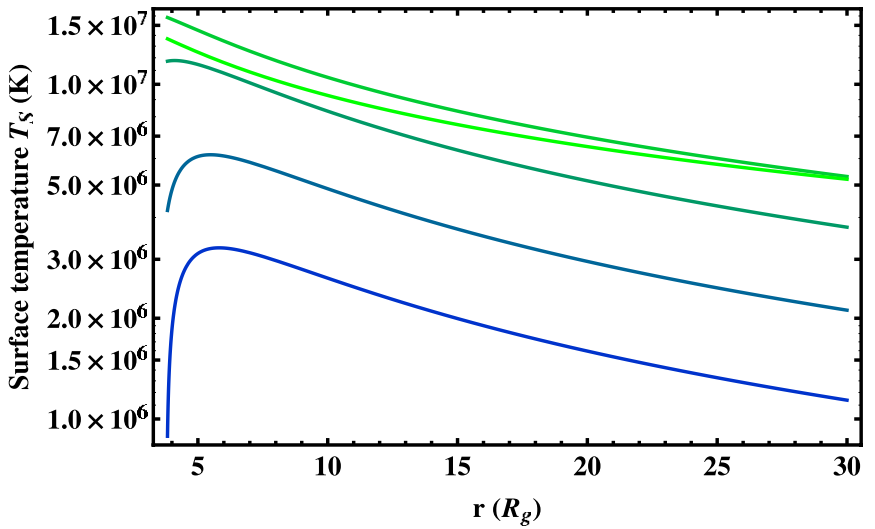

Figure 9. Surface temperature of the slim disk for the case B6Su at various times after the disruption. From top to bottom: at $t=8$ months, $t=2$ months, $t=3$ years, $t=10$ years, and $t=30$ years, respectively. As time progresses, the accretion rate decreases from $\dot{M} \sim 4000 \dot{M}_{\text {Edd }}$ to $\dot{M} \sim 4 \dot{M}_{\text {Edd }}$. The inner disk boundary is chosen to be at $r_{\mathrm{ISCO}}=3.83 R_{\mathrm{g}}$.

(A color version of this figure is available in the online journal.)

opacity, $\tau_{\mathrm{sc}} \gg 1$, is much larger than the bremsstrahlung absorption opacity $\tau_{\text {abs }}$. The electron scattering opacity is

$$
\kappa_{\mathrm{sc}}=\frac{\sigma_{T} n_{e}}{m_{p} n_{\mathrm{nuc}}} \approx 0.2 \mathrm{~cm}^{2} \mathrm{~g}^{-1},
$$

whereas the absorption opacity is

$$
\begin{aligned}
\kappa_{\mathrm{abs}} & =\frac{\left(1-e^{-x}\right) e^{6} h^{2} Z \rho_{c}}{3 c k_{B}^{3} m_{e} m_{p}^{2} T_{s}^{7 / 2} x^{3}} \sqrt{\frac{2 \pi}{k_{B} m_{e}}} \\
& =\frac{4.4 \times 10^{25}\left(1-e^{-x}\right) \rho_{c}}{T_{s}^{7 / 2} x^{3}},
\end{aligned}
$$

where $x=h v /\left(k_{B} T\right)$ (Shapiro \& Teukolsky 1986). The total emitted power through one side of the disk with surface temperature $T_{s}$ is then

$$
F_{-}=\frac{a_{\mathrm{BB}} c}{4} T_{s}^{4} \sqrt{\frac{\bar{\kappa}_{\mathrm{abs}}}{\bar{\kappa}_{\mathrm{sc}}}},
$$

where, in a one-zone model, the central density is taken as a proxy for the surface density. Here $\bar{\kappa}$ denotes mean opacities. We use here the Rosseland mean opacity (Shapiro \& Teukolsky 1986), $\bar{\kappa}_{\text {abs }}=3.2 \times 10^{22} Z \rho_{c} T_{s}^{-7 / 2}$.

The energy flux delivered to the surface is

$$
F_{\text {surf }}=F_{-}=\frac{a_{\mathrm{BB}} c}{3} \frac{T_{c}^{4}}{\tau_{\mathrm{sc}}} .
$$

To sustain the radiation flow through the surface, this energy flux is equal to the radiated flux. Thus, from Equations (28) and (29) we obtain that $T_{s} \propto T_{c}^{16 / 9}$. In a slim disk, cooling does not balance heating, $F_{+} \neq F_{-}$. The residual energy is stored within the accreting material. The energy balance can then be written as

$$
Q=F_{+}-F_{-},
$$

where the residual energy per unit surface area is given as

$$
Q=\Delta U+A=\frac{4}{3} H a_{\mathrm{BB}} T_{c}^{4}\left(3 \frac{1}{T_{c}} \frac{d T_{c}}{d t}-\frac{1}{\rho_{c}} \frac{d \rho_{c}}{d t}\right)
$$

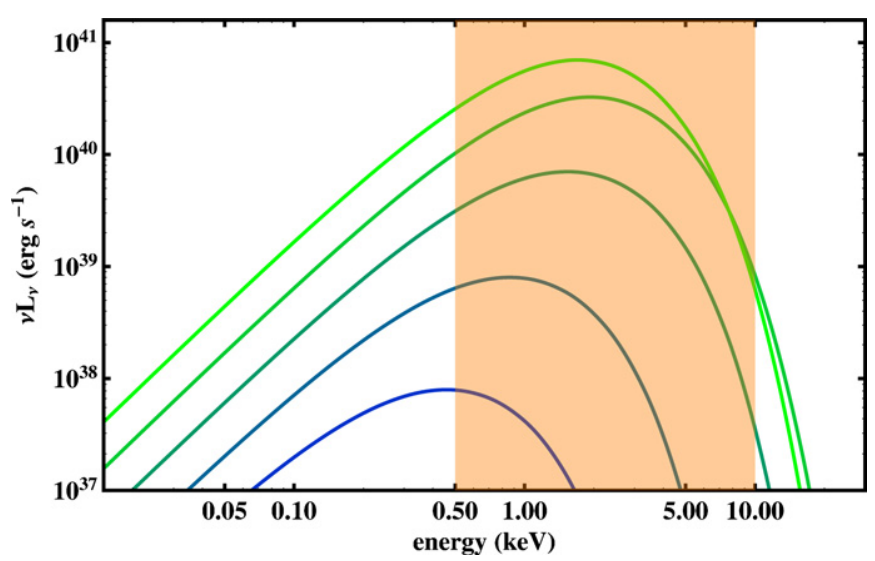

Figure 10. Spectrum for the slim disk formed following the tidal disruption at various times. The spectra shown correspond to the case B6Su as in Figure 9. From top to bottom: at $t=2$ months, $t=8$ months, $t=3$ years, $t=10$ years, and $t=30$ years, respectively. The inner boundary is chosen to be at $r_{\mathrm{ISCO}}=3.83 R_{\mathrm{g}}$. The spectrum gets harder with time, but the luminosity decreases.

(A color version of this figure is available in the online journal.)

where $d t=-d r / v_{r}$. We solve Equations (21) and (22) for the central density $\rho_{c}$. The central temperature $T_{c}$ is obtained from Equation (30) with the help of Equations (20), (29), and (31). Given the central density and temperature, we calculate the surface temperature $T_{s}$ from Equations (28) and (29), as well as the corresponding spectrum.

Figure 9 shows the dependence of surface temperature $T_{s}$ on radius $R$ for the accretion rates inferred from the output of the B6Su simulation. Later times correspond to lower accretion rates. The temperature $T_{s}$ is, in general, higher at higher accretion rates, i.e., earlier times. This is because the energy release per unit time goes up with accretion rate while the radiating surface area stays constant. At very low $\dot{M}$, our slim disk accretion model resembles the standard thin disk model (Shakura \& Sunyaev 1973) so $T_{S} \rightarrow 0$ at the ISCO. In contrast, $T_{s}$ at the ISCO saturates at the highest accretion rates. With little energy release, $F_{+} \gg F_{-}$, the energy density is proportional to the accretion rate $Q=F_{+} \propto M$. In turn, the scattering opacity is also proportional to the accretion rate $\tau_{\mathrm{sc}} \propto n_{e} \propto \dot{M}$. The energy density of radiation is proportional to $T_{c}^{4}$. In addition, there is a factor of $\dot{M}$ in both the numerator and the denominator of the expression (29) for the surface flux. In the end, $F_{\text {surf }} \propto \dot{M}$ in the radiatively inefficient regime.

The corresponding disk spectra are shown in Figure 10. From top to bottom, $v L_{v}$ as a function of energy are shown at 2,8 months, then 3,10 , and 30 years, respectively. Like $T_{s}$, the hard tail of the spectrum also saturates at the highest accretion rates. On the other hand, the low-energy tail does not saturate at $\dot{M} \sim 4000 \dot{M}_{\text {Edd }}$. This is because the radiation time is still shorter than or comparable to the advection timescale at large distances from the $\mathrm{BH}$, where the low-energy tail is emitted. As the accretion rate drops with time, the luminosity (the area under the curve) also drops.

The integrated luminosity is shown in Figure 11 for the case B6Su. At late times, i.e., $t \gtrsim 2$ years, the luminosity is proportional to the accretion rate and decreases as $t^{-5 / 3}$. Specifically, we estimate a luminosity $L \simeq 0.05 \dot{M} c^{2}$ for our disk model. Note that the estimated luminosity in a fully general relativistic model is $L \simeq 0.091 \dot{M} c^{2}$ for a thin disk without radiative transfer effects (Bardeen et al. 1972). At early times $t \lesssim 2$ years, the luminosity saturates at about the 


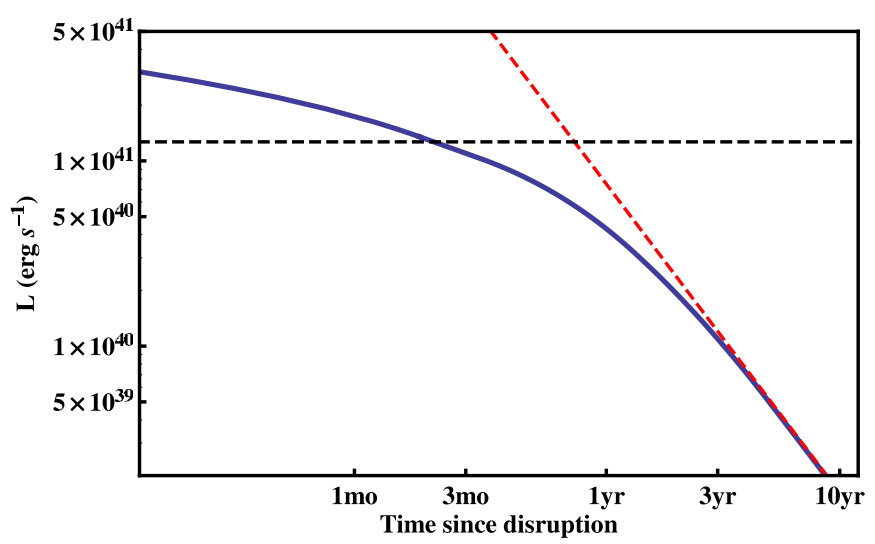

Figure 11. Light curve (solid line) of the slim disk following the tidal disruption in the case $\mathrm{B} 6 \mathrm{Su}$. The inner boundary is chosen to be at $r_{\mathrm{ISCO}}=3.83 R_{\mathrm{g}}$. The luminosity decreases with time, first slowly, then sharply below Eddington (horizontal dashed line). The behavior asymptotes to a power law $L=$ $0.05 \dot{M} c^{2} \propto t^{-5 / 3}$ at low luminosity (inclined dashed line), when all thermal energy is radiated on the way to ISCO. The model cannot be trusted if luminosity significantly exceeds Eddington value.

(A color version of this figure is available in the online journal.)

Eddington value $L_{\text {Edd }}=1.4 \times 10^{41} \mathrm{erg} \mathrm{s}^{-1}$. The luminosity at times $t<2$ months is not reliable, since the slim disk model may not be an adequate representation of accretion at rates $\dot{M}>4000 \dot{M}_{\text {Edd }}$. For instance, when the outflow from the inner disk is present, the luminosity may further increase (Owocki \& Gayley 1997; Shaviv 2001; Begelman 2001; Dotan \& Shaviv 2011). Such a computation is, however, beyond the scope of this paper.

We consider now the radiative properties of disks from the other cases with different penetration parameters $\beta$, spin magnitudes $a^{\star}$, and spin orientations. Figure 12 shows the disk spectra at $t=1 \mathrm{yr}$ after the disruption for all the cases studied. In all cases, we assumed a slim disk with inner boundary at $r_{\mathrm{ISCO}}$ and outer boundary at $30 R_{\mathrm{g}}$.

The spectra in Figure 12 are different because of two factors. The first contributing factor is the accretion rate $\dot{M}$, which we parameterize by the fallback time. More debris falls back onto the $\mathrm{BH}$ in the B6Sa case than in the other cases (see Figure 8). Therefore, the spectrum for the B6Sa case is systematically brighter. Similarly, the lower accretion rate for the B6Si case makes its spectrum systematically weaker than the others. We found that one can achieve coincidence of spectra among all $a^{\star}=0.6$ cases with a time shift. That is, at times when the accretion rates $\dot{M}$ are identical for different simulations, the spectra are also identical. The spectrum for the B6Sa case at about $t=16$ months is identical to the spectrum for the B6Su case at $t=12$ months and to the spectrum from the B6Si case at about $t=9$ months. Also, more matter is swallowed by the $\mathrm{BH}$ initially in the B8Sa case when compared with the B6Sa case, but at later times, less matter that will be eventually accreted is scattered. Thus, the fallback time is lower for the B8Sa case, resulting in a less luminous spectrum. The spectra also vary because of changes in the ISCO radius. For instance, the ISCO is farther from the $\mathrm{BH}$ in the B6S0 case with spin $a^{\star}=0$. Since little energy release is taking place inside $r_{\mathrm{ISCO}}=6 R_{\mathrm{g}}$, its spectrum is significantly softer.

\subsection{Obscuration}

The edge-on view of a slim disk is self-obscured. The spectrum of a self-obscured disk is not easy to determine. We can

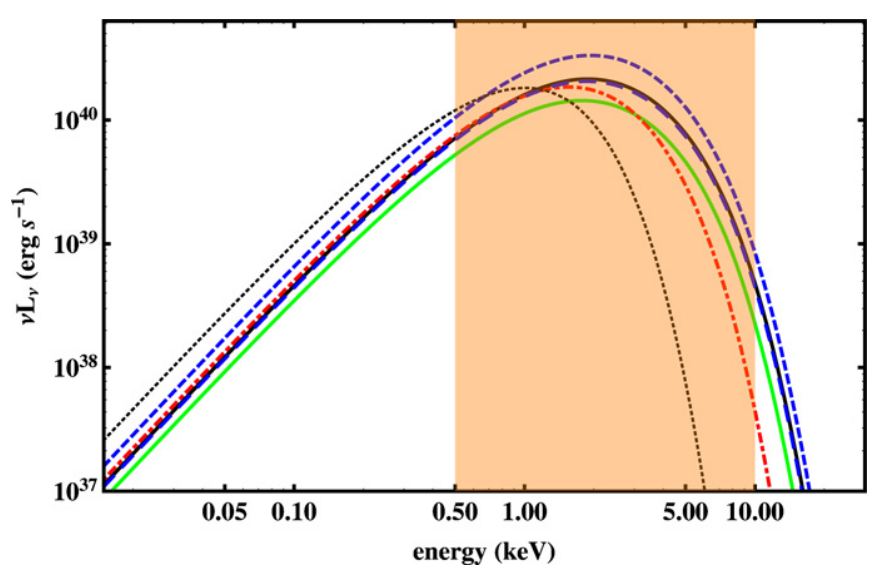

Figure 12. Spectra of our slim disk models at $t=1 \mathrm{yr}$ : B6Su case (top solid/black), B6Si case (bottom solid/green), B6Sa case (short-dashed/blue), B8Sa case (long-dashed/blue), and B6S0 case (dot-dashed/red). Differences are due to changing spin and fallback time. Dotted line corresponds to an edgeon view of a slim disk for b6ap6 simulation. The edge-on disk has a softer spectrum and a comparable luminosity.

(A color version of this figure is available in the online journal.)

approximate the spectrum by assuming that the thick outer edge at $r=30 R_{\mathrm{g}}$ emits modified blackbody radiation the way the top of the slim disk does. The corresponding edge-on spectrum is included in Figure 12 as the black dotted line. It is softer, since only the material far from the $\mathrm{BH}$ contributes. The surface area of the thick outer edge is large, though, resulting in a raised low-energy tail.

Besides exploring the properties of a slim disk, we investigated whether the emission from the disk is visible through the outflowing debris surrounding the disk. Figure 13 shows the $(\cos \theta, \phi)$ directions in which the debris is scattered in the "sky" from the $\mathrm{BH}$ point of view. We considered only the material outside a sphere of radius $150 R_{\mathrm{g}}$, corresponding to the boundary between the bound and the unbound matter at time $t \approx 6 \mathrm{~s}$ after the disruption. The unbound matter travels essentially along straight lines at $t \approx 6 \mathrm{~s}$, so Figure 13 adequately represents the angular distribution of matter at late times. White color in Figure 13 indicates that there is no matter traveling in that direction, whereas all darker colors indicate the presence of gas.

The optical depth is very high at directions that are not denoted by white. The inner disk would be completely obscured along those directions. The left panel in Figure 13 shows the distribution of the scattered debris for the B6Si case. Note that although the debris is scattered all over the sky, there are some preferential directions manifest as a sinusoid in the $(\cos \theta, \phi)$ plane. On the other hand, in the case B6Su shown on the right panel, the debris is scattered preferentially in the disruption plane. Thus, the orientation of BH spin determines the direction of debris outflow for ultra-close encounters.

We consider now the situation in which we view a WD-BH system with an unknown orientation. Recall that we have assumed that the disk at late times is fully aligned with the initial disruption plane. As discussed before, the disk can, in principle, be viewed both edge-on or face-on, although the edge-on disk is self-obscured and has a peculiar spectrum (see Figure 12). The probability that the disk is obscured by the outflowing debris is proportional to the solid angle subtended by the debris. This solid angle corresponds to the normalized surface area on the $(\cos \theta, \phi)$ plane (see Figure 13). There may in principle be four different types of obscuration: (1) edge-on self-obscured disk also obscured by outflowing debris, (2) edge-on self-obscured 

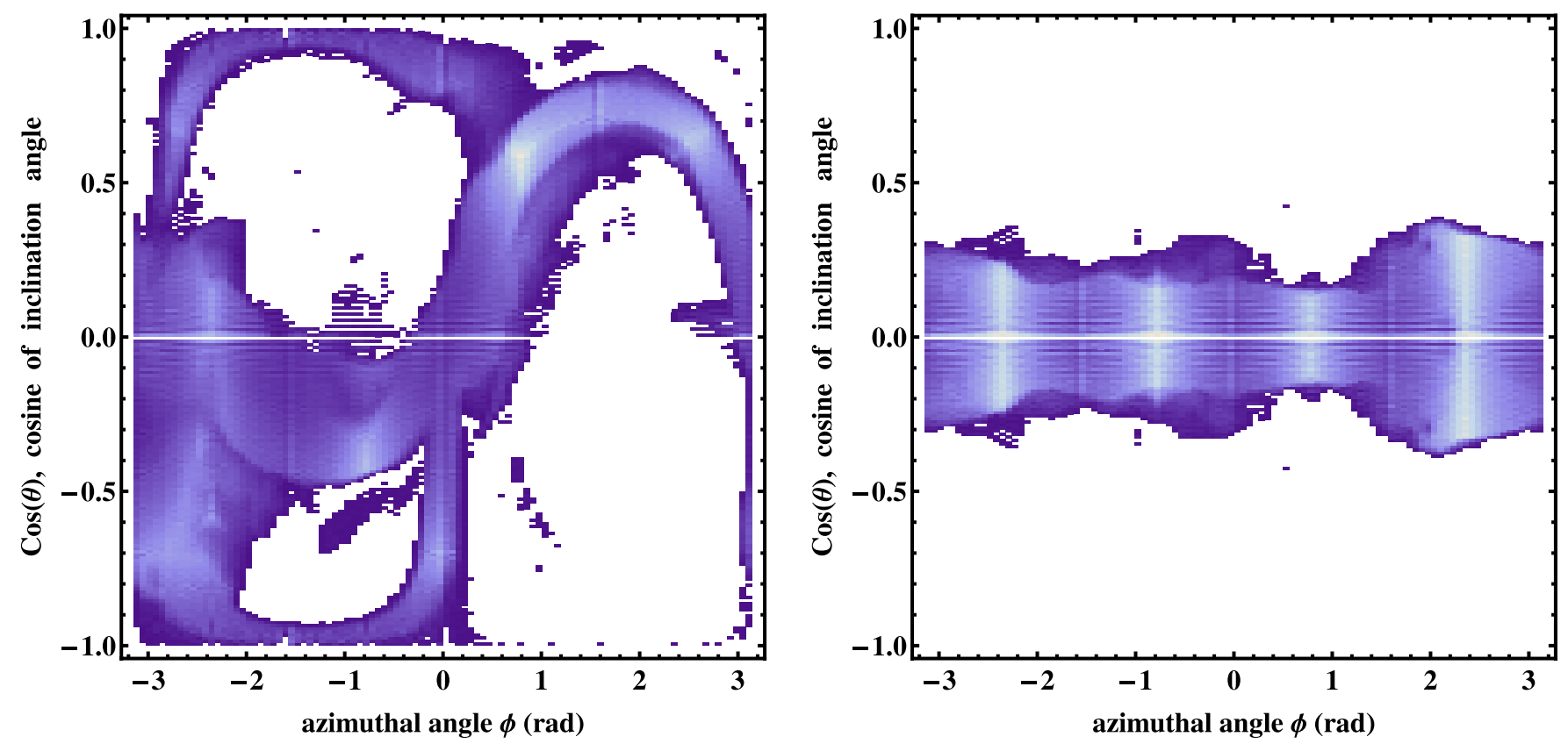

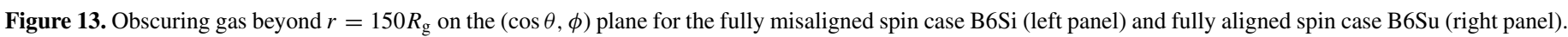

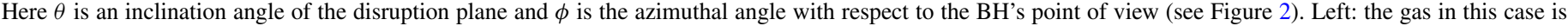

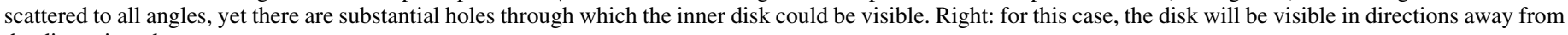
the disruption plane.

(A color version of this figure is available in the online journal.)

disk unobscured by outflowing debris, (3) face-on disk obscured by outflowing debris, and (4) face-on completely unobscured disk.

When the height of the photosphere equals the disk height $H=r$ (inclination $\theta=45^{\circ}$ ), the probability to encounter a self-obscured edge-on disk is $p_{\text {edge }}=71 \%$. The obscuration probability by outflowing debris is depicted in Figure 14 for both edge-on and face-on disks. The panels show the probability as a function of debris location at $t=6 \mathrm{~s}$ after the disruption. At that time, the boundary between the bound and the unbound matter is located at $150 R_{\mathrm{g}}$. Thus, this obscuration probability is also the obscuration probability $p_{\text {obs }}$ for days to years later. The obscuration probability $p_{\text {obsledge }}$ of an edge-on disk can be inferred from the left panel. Since most debris is scattered in the disruption plane, this probability is relatively high. The values reach $p_{\text {obs|edge }} \approx 50 \%$, practically independent of spin value, spin orientation, or penetration factor. The obscuration probability of a face-on disk $p_{\text {obs|face }}$ can be inferred from the right panel. Only the fully misaligned case B6Si scatters significant amount of debris perpendicular to the disruption plane, which leads to relatively high $p_{\text {obs|face }} \approx 40 \%$ for this simulation. Partially and fully aligned simulations all scatter matter within $45^{\circ}$ from the disruption plane and thus cannot obscure the face-on disk, so that $p_{\text {obs|face }}=0 \%$. The total obscuration probability is

$$
p_{\text {obs }}=p_{\text {obs|face }} p_{\text {face }}+p_{\text {obs|edge }}\left(1-p_{\text {face }}\right) \text {. }
$$

The values are $p_{\text {obs }} \approx 50 \%$ for the $\mathrm{B} 6 \mathrm{Si}$ case and $p_{\mathrm{obs}} \approx 30 \%$ for the B6Su, B6Sa, B8Sa, and B6S0 cases. The misaligned simulation with $\beta=8$ yields a marginally higher $p_{\text {obs edge }}=$ $52 \%$ compared to a similar simulation with $\beta=6$ for which $p_{\text {obs|edge }}=44 \%$. As expected, deeper penetration results in wider scattering of debris. However, surprisingly, partially aligned and fully aligned simulations with $\beta=6$ and $a^{\star}=$ 0.6 yield almost the same obscuration probabilities.
We have previously stated that if there is debris along the line of sight, then the inner disk is obscured. However, the optical depth of debris decays as the debris expands and eventually becomes transparent. Let us estimate the time $t_{\text {trans }}$ when the optical depth of X-ray absorption equals unity $\tau_{\mathrm{abs}}=1$. The actual time will of course depend on the line of sight. The debris consists mostly of neutral carbon and oxygen with high absorption cross-section $\sigma_{\text {abs }} \simeq 2 \times 10^{-20} \mathrm{~cm}^{-2}$ within $0.5-10 \mathrm{keV}$ photon energy range (Henke et al. 1993). The typical outflow velocity of the debris is a substantial fraction of the speed of light $v_{\text {out }} \approx 0.15 c$. The transparency time is then

$$
t_{\text {trans }}=\sqrt{\frac{f_{\mathrm{unb}} M_{\mathrm{wd}} \sigma_{\mathrm{abs}}}{56 \pi m_{p} p_{\mathrm{obs}} v_{\mathrm{out}}^{2}}},
$$

where $f_{\text {unb }}$ is the fraction of outbound material. From our simulations, we found that $f_{\text {unb }} \simeq 22 \%\left(f_{\text {unb }} \simeq 60 \%\right)$ for the B6Si (B6Su) case. With our obscuration probability estimate $p_{\text {obs }} \simeq 50 \%\left(p_{\text {obs }} \simeq 30 \%\right)$ for the B6Si (B6Su) case, we find that $t_{\text {trans }} \approx 2 \mathrm{yr}\left(t_{\text {trans }} \approx 4 \mathrm{yr}\right)$. The transparency time given by Equation (33) is an underestimate, since marginally unbound matter moves at speeds lower than the average $v_{\text {out }}$.

\section{GRAVITATIONAL WAVE EMISSION}

In addition to producing the electromagnetic signatures discussed in previous sections, tidal disruption events are potential sources of $\mathrm{GW}$ radiation. A disruption event generates a burst of GWs lasting $t \sim R_{\mathrm{p}} / V_{\mathrm{p}} \sim\left(R_{\mathrm{p}}^{3} / G M_{\mathrm{bh}}\right)^{1 / 2}$ (Kobayashi et al. 2004; Rosswog et al. 2009). The GW burst produced will have a characteristic frequency $f \sim\left(G M_{\mathrm{bh}} / R_{\mathrm{p}}^{3}\right)^{1 / 2}$ and amplitude $h \sim\left(G M_{\mathrm{wd}} R_{\mathrm{g}}\right) /\left(c^{2} R_{\mathrm{p}} D\right)$, with $D$ the distance to the BH. For 

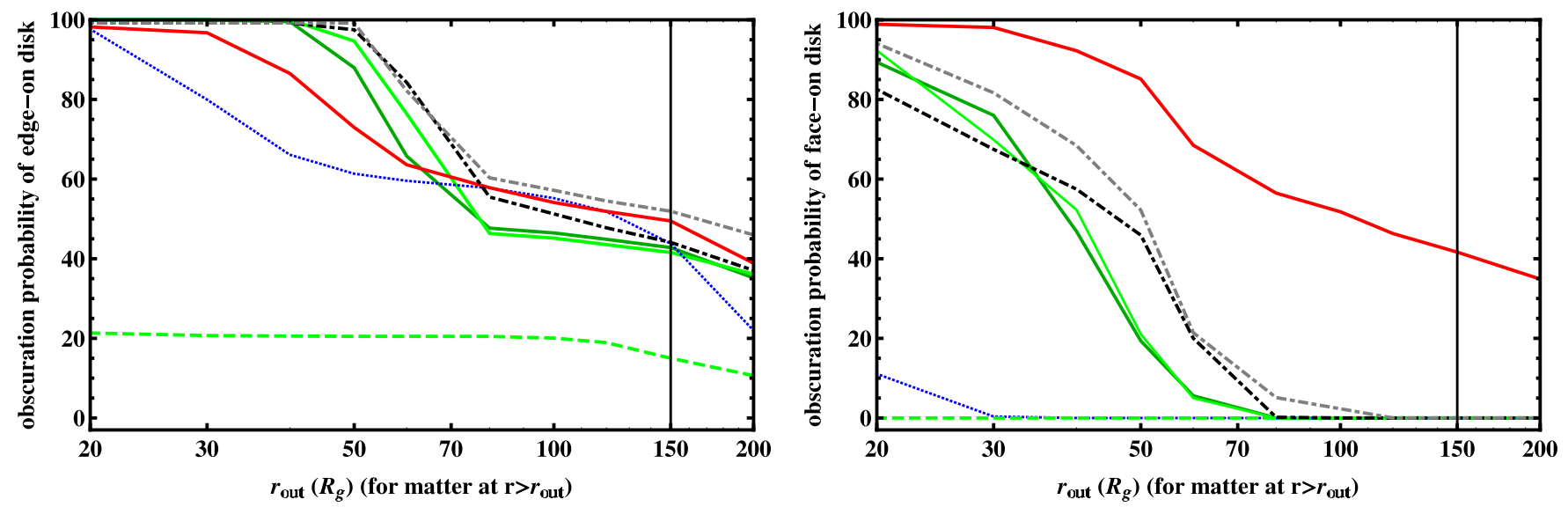

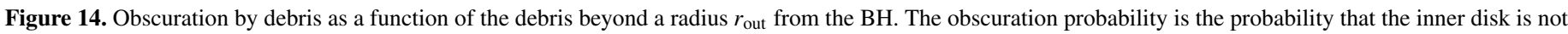

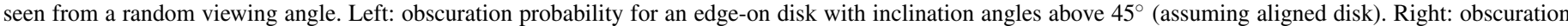

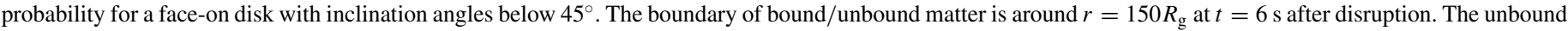

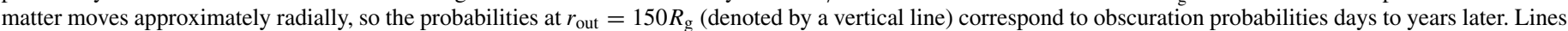

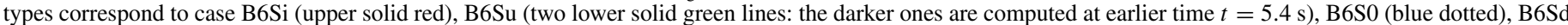

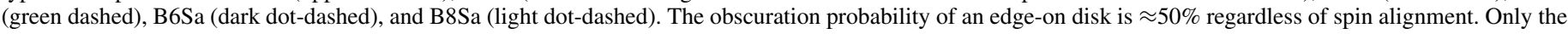

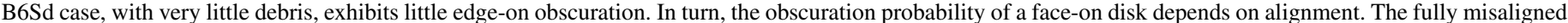
B6Si case is obscured at $50 \%$ for nearly face-on disk orientations, whereas the partially misaligned and aligned cases exhibit no obscuration of a face-on disk.

(A color version of this figure is available in the online journal.)

the case of a WD disrupted by an IMBH (Rosswog et al. 2009),

$$
\begin{gathered}
f \sim 0.68 \beta^{3 / 2}\left(\frac{R_{\mathrm{wd}}}{0.95 R_{\oplus}}\right)^{-3 / 2}\left(\frac{M_{\mathrm{wd}}}{1 M_{\odot}}\right)^{1 / 2} \mathrm{~Hz} \\
h \sim 5.4 \times 10^{-20} \beta\left(\frac{D}{20 \mathrm{kpc}}\right)^{-1}\left(\frac{M_{\mathrm{wd}}}{1 M_{\odot}}\right)^{4 / 3} \\
\times\left(\frac{R_{\mathrm{wd}}}{0.95 R_{\oplus}}\right)^{-1}\left(\frac{M_{\mathrm{BH}}}{10^{3} M_{\odot}}\right)^{2 / 3} .
\end{gathered}
$$

These estimates place the GWs from these events at the lowfrequency edge of the advanced LIGO design sensitivity range. At this edge, the equivalent strain noise is $\sim 10^{-22} \mathrm{~Hz}^{-1 / 2}$ (Shoemaker 2009).

In Figure 15, we compare the GW strain extracted from the spacetime (solid red) to that obtained using the quadrupole formula (Rosswog et al. 2009) applied to the WD's centerof-mass trajectory (dashed blue) for B6Sa. While the qualitative behavior of the two polarizations, $h_{+}$(top) and $h_{x}$ (middle), is similar, the fully nonlinear GW strain differs in both amplitude and phase.

In Figure 16, we show the GW strain as a function of time for disruption events at a distance of $20 \mathrm{kpc}$. Depicted are the two polarizations of GWs: $h_{+}$(solid red line) and $h_{\times}$(dashed green line) for cases B6Sa (top) and B8Sa (bottom). Time $t=0$ denotes approximately the time when the disruption takes place. Evident is the burst-like nature of the GWs. Note that both cases exhibit comparable burst duration and strain amplitude. This is expected since the only difference between B6Sa and B8Sa is their value of $\beta$. Although we have stated that case $\mathrm{B} 6 \mathrm{Sa}$ has $\beta=6$ and $\mathrm{B} 8 \mathrm{Sa}$ has $\beta=8$, from Table 2, we have that the actual penetration factor for both cases is $\beta^{*} \simeq 10$. Interestingly, the other cases also showed comparable burst durations, $\sim 4 \mathrm{~s}$, suggesting a weak dependence with the orientation of the spin and penetration factor.

Figure 17 shows the power spectrum for all the cases investigated where we assume that the events took place at a distance of

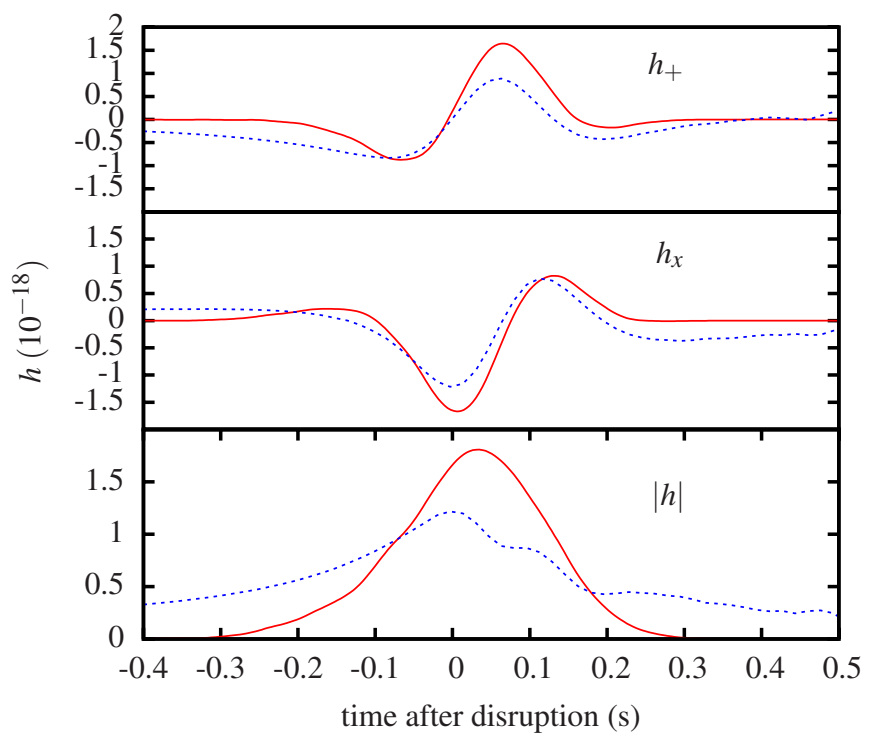

Figure 15. Gravitational wave strain as calculated in full general relativity (solid red) and from applying the quadrupole formula (dashed blue) for B6Sa at a distance of $20 \mathrm{kpc}$. Depicted are the two polarizations, $h_{+}$(top) and $h_{\times}$ (middle), as well as the amplitude (bottom).

(A color version of this figure is available in the online journal.)

$20 \mathrm{kpc}$. All cases indicate a characteristic frequency of $\sim 3.2 \mathrm{~Hz}$. This is about an order of magnitude smaller than the estimated frequency using Equation (34) with $\beta^{*} \simeq 9$, the average real penetration factor (see Table 2). This should not be too surprising since rough estimates using Equations (34) and (35) do not take into account, among other things, spin effects. Similarly, Figure 17 shows characteristic strain amplitudes of $\sim 10^{-18}$, an order of magnitude higher than the estimate using Equation (35) with $\beta^{*} \simeq 9$.

At low frequencies, $\sim 1 \mathrm{~Hz}$, we note that the B6Sa and B6Su cases in particular have higher strain amplitude than the rest. Interestingly, these are the same cases that, as seen in Figure 7, exhibit significantly lower prompt accretion in the 


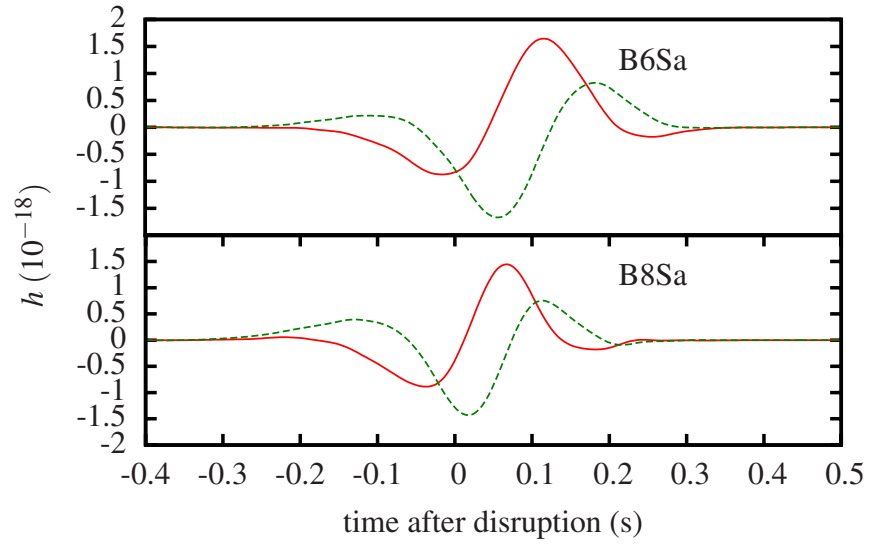

Figure 16. Gravitational wave strain $h$ from disruption events at a distance of $20 \mathrm{kpc}$. Depicted are the two polarizations, $h_{+}$(solid red line) and $h_{\times}$(dashed green line) for cases B6Sa (top) and B8Sa (bottom), respectively.

(A color version of this figure is available in the online journal.)

first second after disruption and higher accretion in the $\sim 1-2 \mathrm{~s}$ after disruption. The third case with higher prompt accretion in the $\sim 1-2$ s interval, B8Sa, however, exhibits the lowest lowfrequency tail. This suggests that relativistic effects create an entangled parameter space involving spin and penetration factor during the disruption phase. Given that the actual penetration factors in all the cases were comparable, $7.6 \leqslant \beta^{*} \leqslant 11$, further study is required to explore this parameter space.

Synergistic observations of electromagnetic emission from the prompt accretion discussed in Section 4, together with GW detections, may provide ways to measure the spin of the $\mathrm{BH}$ since in both instances the emission seems to depend, although not strongly, on the spin orientation. We are currently expanding the parameter space covered by our simulations to investigate this dependence in more detail. Multi-messenger observations will be, of course, only possible if the prompt electromagnetic emission is not obscured by the debris surrounding the $\mathrm{BH}$. If such obscuration prevents observing prompt accretion, the detection of a GW burst would, in that case, still serve as an identifying precursor to the observations of the late fallback electromagnetic emission.

\section{RATES AND OBSERVATIONAL PROSPECTS}

\subsection{Tidal Disruption Rates}

The disruptions of stars by BHs are rare events. More studied are tidal disruptions of stars by SMBHs in galactic centers. Their rate $10^{-5} \mathrm{yr}^{-1}$ per galaxy was predicted theoretically by Magorrian \& Tremaine (1999) and Wang \& Merritt (2004), and found consistent with observations by ROSAT (Donley et al. 2002), XMM-Newton (Esquej et al. 2007), and Galaxy Evolution Explorer (Gezari 2010). The volume density of SMBHs is about (Tundo et al. 2007)

$$
n_{\mathrm{SMBH}}=10^{-2} \mathrm{Mpc}^{-3},
$$

which leads to tidal disruption rate per unit volume

$$
R_{\mathrm{SMBH}}=10^{2} \mathrm{yr}^{-1} \mathrm{Gpc}^{-3} .
$$

The rate of disruption of stars in GCs is much less certain. Baumgardt et al. (2004) predict that, for a GC with $10^{3} M_{\odot}$ $\mathrm{BH}$, the optimistic disruption rate of stars is $10^{-7} \mathrm{yr}^{-1}$ per GC. According to McLaughlin (1999), the mean GC mass is

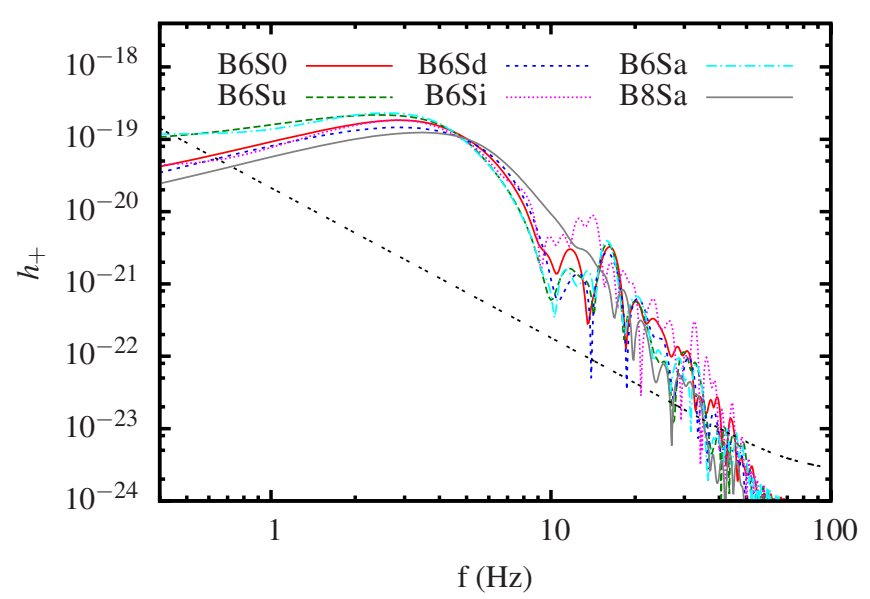

Figure 17. Power spectrum of the $h_{+}$polarization of GWs for all considered cases. We include the advanced LIGO design sensitivity (double-dashed line) for comparison. All cases indicate a characteristic frequency of $3.2 \mathrm{~Hz}$. At low frequencies, $\sim 1 \mathrm{~Hz}$, there is a hint of a dependence of the amplitude on the spin orientation.

(A color version of this figure is available in the online journal.)

$M_{\mathrm{GC}}=2.4 \times 10^{5} M_{\odot}$, which is larger than clusters considered in Baumgardt et al. (2004). For the largest cluster simulated by Baumgardt et al. (2004) with $N=131,072$ stars they find $15 \%$ of disrupted stars to be WDs. Therefore, the optimistic rate of WD-IMBH tidal disruptions is

$$
D=1.5 \times 10^{-8} \mathrm{yr}^{-1}
$$

per cluster. The number of GCs changes dramatically from one galaxy to another (Harris 1991). However, GCs have about constant formation efficiency $\epsilon_{\mathrm{cl}}=0.0026$ (McLaughlin 1999), which is the ratio of GC mass to the initial mass of gas in a galaxy. We assume half of baryonic matter in galaxies, take the Hubble constant $H_{0}=70.4 \mathrm{~km} \mathrm{~s}^{-1} \mathrm{Mpc}^{-1}$ and current baryonic content $\Omega_{b}=0.00456$ (Komatsu et al. 2011), and find the number density of GCs as

$$
n_{\mathrm{GC}}=0.5 \frac{3 H_{0}^{2}}{8 \pi G} \frac{\Omega_{b} \epsilon_{\mathrm{cl}}}{M_{\mathrm{GC}}}=34 \mathrm{Mpc}^{-3}
$$

This density overpredicts the number of GCs in the Milky Way but is consistent with the large population of GCs in elliptical galaxies such as M87. It is not known what percentage of GCs contain an IMBH. For optimistic estimates, we take one $10^{3} M_{\odot}$ IMBH per cluster. Then, the disruption rate per unit volume is

$$
R_{\mathrm{WD}-\mathrm{IMBH}}=500 \mathrm{yr}^{-1} \mathrm{Gpc}^{-3} \text {. }
$$

This rate is even higher than $R_{\mathrm{SMBH}}$, but the WD-IMBH disruptions are much less luminous, so they are harder to find. Also, the rates of tidal disruptions in GCs are quite uncertain (Baumgardt et al. 2004), so even disproving the rate given by Equation (40) does not necessarily disprove the hypothesis that every GC has an IMBH.

Out of all tidal disruptions, we focused on ultra-close ones with the ratio of tidal radius to pericenter distance about $\beta=6$. These constitute a fraction of all disruptions. For a realistic triaxial potential of a GC, chaotic feeding would prevail (Merritt \& Poon 2004). Chaotic feeding leads to tidal disruption probability proportional to $R_{p}$ for objects coming within pericenter distance $R_{p}$ as a clear consequence of gravitational focusing. Therefore, tidal disruptions with $\beta>5$ constitute $1 / 5$ of all events. The 
correspondent optimistic rate is

$$
R_{\mathrm{WD}-\mathrm{IMBH}, \beta>5}=100 \mathrm{yr}^{-1} \mathrm{Gpc}^{-3} \text {. }
$$

\subsection{Observations of X-Ray Flares}

Considered ultra-close tidal disruptions manifest with a yearlong X-ray flare at an Eddington luminosity off the center of the galaxy. Tidal disruptions by SMBHs rather produce an optical flare (Strubbe \& Quataert 2009) in the center of the galaxy. Let us explore if the optimistic high rates of tidal disruptions are consistent with past observations and if future observations have a chance of catching such an event.

The ROSAT survey probed all sky down to $F_{R O S A T}=$ $4 \times 10^{-13} \mathrm{erg} \mathrm{s}^{-1} \mathrm{~cm}^{-2}$ flux within $0.1-2.4 \mathrm{keV}$ band (Voges et al. 2000). The peak of X-ray tidal disruption spectrum at $1 \mathrm{yr}$ (see Figure 12) is at about $2 \mathrm{keV}$, so that more than half of photons are captured. Taking luminosity $L=4.3 \times 10^{40} \mathrm{erg} \mathrm{s}^{-1}$ of $\mathrm{B} 6 \mathrm{Su}$ simulation at $t=1 \mathrm{yr}$, we obtain the maximum distance of

$$
d_{\text {ROSAT }}=25 \mathrm{Mpc} .
$$

Therefore, the expected number of observed WD-IMBH disruptions is $N_{R O S A T}=0.015$, which is consistent with no events. With Chandra, 100 photons can be obtained over $30 \mathrm{ks}$ from the source $200 \mathrm{Mpc}$ away and over $1 \mathrm{Ms}$ from the source $1.2 \mathrm{Gpc}$ away, according to the Portable, Interactive Multi-Mission Simulator (Mukai 2000). However, owing to a small field of view of the satellite, the careful choice of field is required to observe at least one event.

In turn, future missions like Wide Field X-ray Telescope (Conconi et al. 2010) can detect a substantial number of events. It is much more sensitive than ROSAT and observes in the same waveband. The flux limits of $3 \times 10^{-17}, 5 \times 10^{-16}$, and $3 \times 10^{-15}$ for deep, medium, and wide surveys with correspondent solid angles 100, 3000, and 20,000 $\mathrm{deg}^{2}$ yield maximum event distances $2.5 \mathrm{Gpc}, 600 \mathrm{Mpc}$, and $245 \mathrm{Mpc}$. This translates into 76,33 , and 16 WD-IMBH disruptions, or five times fewer ultraclose disruptions.

Tidal disruptions can also produce powerful prompt superEddington X-ray flare with the duration of minutes in addition to the Eddington-limited X-ray flux on the timescale of $\sim 1 \mathrm{yr}$. The light curve, spectrum, and detection prospects will be discussed in the next paper (R. V. Shcherbakov et al. 2012, in preparation).

\section{DISCUSSION AND CONCLUSIONS}

Our work addresses disruptions of WDs by IMBHs. This is the first fully general-relativistic study that includes both strong gravity (the metric of the spinning $\mathrm{BH}$ ) and dynamical gravity effects (GWs). We focused on ultra-close disruptions, where the periapsis radius is comparable to the $\mathrm{BH}$ gravitational radius. Our study presents for the first time results on the influence of the BH spin on the disruption.

Our study made some simplifying assumptions. Instead of employing a degenerate equation of state for the WD, we used an ideal gas law. This is, however, expected to have a very minor effect on dynamics. If the entropy is approximately constant, the evolution of a degeneracy-pressure-supported star is identical to that with an ideal gas pressure. The choice of pressure prescription does not matter until shocks and turbulence take over. Similarly, the absence of explicit radiation pressure does not influence the initial stages of the disruption. Cold outflowing debris may not have significant radiation pressure support, and only the fallback disk will be radiation pressure dominated. Radiation emitted from the inner disk may halt the infall of the marginally bound debris. However, the disk is self-obscured near the disruption plane where most debris is scattered, and the photon mean free path is quite small. The photons thus cannot effectively transfer energy from the inner flow to the outer flow to unbind the debris. Should radiation pressure be included, the same internal energy contributes a radiation pressure smaller than the gas pressure, and thus the total pressure in the medium would be lower.

Our study does not include nuclear reactions, believed to be important for deep WD disruptions (Rosswog et al. 2009). Temperature-density tracks of the matter in our simulations as depicted in Figure 4 are comparable to those found in Figure 14 of Rosswog et al. (2009). The tracks suggest that nuclear burning of carbon and oxygen should occur in the cases we have studied. The additional energy gained in $\mathrm{C}$ and $\mathrm{O}$ burning into $\mathrm{Fe}$ is 1 $\mathrm{MeV}$ per nucleon (Dursi \& Timmes 2006). This translates to a gain of energy of $\sim 0.001 M c^{2}$. On the other hand, the descent into the gravitational potential of the $\mathrm{BH}$ yields energies of $\sim 0.1 M c^{2}$ for a periapsis radius of $R_{\mathrm{p}} \sim 10 R_{\mathrm{g}}$. The dispersion of debris energies after ultra-close disruptions is also large. Thus, the extra $0.001 M c^{2}$ energy from the burning will not likely have a dramatic effect on the debris. The optical light curve may indeed change after an initially cold WD is heated by nuclear reactions, but the influence on the fallback disk is expected to be small.

A realistic fallback disk simulation should incorporate magnetic fields. Magnetic fields give rise to large viscosity and allow the disk to self-consistently accrete. The assumed parameter $\alpha$ will be a proxy for viscosity until magnetized simulations can be run for a sufficiently long time. The presence of magnetic fields may readily lead to an outflow (Blandford \& Payne 1982), which may boost luminosity far above the Eddington limit, or radiation pressure itself may drive an outflow under certain circumstances (Shaviv 2001). The duration of our simulations ( $\sim 7 \mathrm{~s})$ was too limited to directly model the fallback. The temporal dependence of accretion rate depicted in Figure 8 is a ballistic guess based on a confirmed flat distribution of mass over energy. Any outflow or halted inflow at late times may result in deviations of the fallback law from $\dot{M} \propto t^{-5 / 3}$.

We make a distinction in the paper between the face-on and edge-on disks. In reality, only minor differences between these orientations may exist. The disk with extreme accretion rate may drive a wind or have a corona, which might completely obscure the very inner portions of the disk. In such a case, the spectrum would be similar to an edge-on spectrum regardless of orientation. The precise quantitative predictions of spectra will only be possible with detailed numerical simulations of the fallback accretion.

Our study should be viewed as a step toward realistic tidal disruptions of WDs by IMBHs. Some of the conclusions may hold up despite our limited understanding of matter fallback and super-Eddington accretion flows. Our main conclusions are as follows:

1. For a non-spinning $\mathrm{BH}$ and a $\mathrm{BH}$ with a spin aligned or misaligned with the orbital angular momentum, the debris after disruption forms a thick accretion disk.

2. For misaligned spins, frame-dragging effects scatter the debris around the $\mathrm{BH}$ and will often obscure the inner region from observation.

3. There is a qualitatively different behavior before and after a timescale of $\sim 1 \mathrm{yr}$ when the accretion rate approaches 
Eddington accretion rate. The accretion flow luminosity stays around $L_{\mathrm{Edd}}$ before $\sim 1 \mathrm{yr}$ and starts dropping as $t^{-5 / 3}$ afterward.

4. The spectrum peaks at soft X-rays. The spectra are similar to thin disk spectra at low accretion rates $\dot{M} \ll \dot{M}_{\text {Edd }}$.

5. Self-obscuration and obscuration by debris are present in the system. Self-obscuration leads to softer spectrum, while obscuration by debris may make a fallback disk invisible during most of the active accretion period.

6. The GW signal depends weakly on the orientation of the spin. The GW burst will be challenging to be detected for extragalactic sources.

The results reported in this paper were obtained with the MAYA code of the numerical relativity group at Georgia Tech. The MAYA code solves the Einstein equations of general relativity using the Baumgarte-Shapiro-Nakamura-Oohara-Kojima formulation, and BHs are modeled following the moving puncture method (Campanelli et al. 2006; Baker et al. 2006). The source code is partially generated using KRANC (Husa et al. 2006). The Maya code works under CaCtUs, with adaptive mesh refinement provided by CARPET (Schnetter et al. 2004). Hydrodynamics in the MAYA code is based on the public version of the WHISKY code developed by the European Union Network on Sources of Gravitational Radiation (Baiotti et al. 2005). We construct initial data using the 2Punctures code of Ansorg et al. (2004) and use AHFinderDiRect (Thornburg 2004) to locate the apparent horizon. Both codes are part of the Einstein Toolkit (EinsteinToolkit 2011). Details of the MaYA code can be found in Vaishnav et al. (2007), Hinder et al. (2008), Herrmann et al. (2007a, 2007b), and Bode et al. (2008, 2009, 2012).

The authors are thankful to Ramesh Narayan for fruitful discussions. R.V.S. is supported by NASA Hubble Fellowship grant HST-HF-51298.01. R.H. gratefully acknowledges support by the Natural Sciences and Engineering Council of Canada. This work is supported by NSF grants 0653443, 0855892, 0914553, 0941417, 0903973, and 0955825. The computations are held at Teragrid TG-MCA08X009 and the Georgia Tech FoRCE cluster.

\section{REFERENCES}

Abramowicz, M. A., Czerny, B., Lasota, J. P., \& Szuszkiewicz, E. 1988, ApJ, 332,646

Alcubierre, M. 2008, Introduction to 3+1 Numerical Relativity (Oxford: Oxford Univ. Press)

Ansorg, M., Brügmann, B., \& Tichy, W. 2004, Phys. Rev. D, 70, 064011

Baiotti, L., Hawke, I., Montero, P. J., et al. 2005, Phys. Rev. D, 71, 024035

Baker, J. G., Centrella, J., Choi, D.-I., Koppitz, M., \& van Meter, J. 2006, Phys. Rev. Lett., 96, 111102

Bardeen, J. M., \& Petterson, J. A. 1975, ApJ, 195, L65

Bardeen, J. M., Press, W. H., \& Teukolsky, S. A. 1972, ApJ, 178, 347

Baumgardt, H., Makino, J., \& Ebisuzaki, T. 2004, ApJ, 613, 1143

Baumgarte, T. W., \& Shapiro, S. L. 2010, Numerical Relativity: Solving Einstein's Equations on the Computer (Cambridge: Cambridge Univ. Press) Begelman, M. C. 2001, ApJ, 643, 1065

Blandford, R. D., \& Payne, D. G. 1982, MNRAS, 199, 883

Bode, T., Bogdanovic, T., Haas, R., et al. 2012, ApJ, 744, 45

Bode, T., Laguna, P., Shoemaker, D. M., et al. 2009, Phys. Rev. D, 80, 024008

Bode, T., Shoemaker, D., Herrmann, F., \& Hinder, I. 2008, Phys. Rev. D, 77, 044027

Bogdanović, T., Eracleous, M., Mahadevan, S., Sigurdsson, S., \& Laguna, P. 2004, ApJ, 610, 707

Bowen, J. M., \& York, J. W., Jr. 1980, Phys. Rev. D, 21, 2047

Campanelli, M., Lousto, C. O., Marronetti, P., \& Zlochower, Y. 2006, Phys. Rev. Lett., 96, 111101

Cannizzo, J. K., Lee, H. M., \& Goodman, J. 1990, ApJ, 351, 38

Carter, B., \& Luminet, J. P. 1982, Nature, 296, 211
Clausen, D., \& Eracleous, M. 2011, ApJ, 726, 34

Conconi, P., Campana, S., Tagliaferri, G., et al. 2010, MNRAS, 405, 877

Czerny, B., \& Elvis, M. 1987, ApJ, 321, 305

Davis, S. W., Narayan, R., Zhu, Y., et al. 2011, ApJ, 734, 111

Done, C., \& Davis, S. W. 2008, ApJ, 683, 389

Donley, J. L., Brandt, W. N., Eracleous, M., \& Boller, T. 2002, AJ, 124, 1308

Dotan, C., \& Shaviv, N. J. 2011, MNRAS, 413, 1623

Dursi, L. J., \& Timmes, F. X. 2006, ApJ, 641, 1071

Eddington, A. S. 1926, The Internal Constitution of the Stars (Cambridge: Cambridge Univ. Press)

EinsteinToolkit 2011, Einstein Toolkit: Open Software for Relativistic Astrophysics

Esquej, P., Saxton, R. D., Freyberg, M. J., et al. 2007, A\&A, 462, L49

Evans, C. R., Iben, I., \& Smarr, L. 1987, ApJ, 323, 129

Evans, C. R., \& Kochanek, C. S. 1989, ApJ, 346, L13

Farrell, S., Webb, N., Barret, D., Godet, O., \& Rodrigues, J. 2009, Nature, 460, 73

Fragile, P. C., Blaes, O. M., Anninos, P., \& Salmonson, J. D. 2007, ApJ, 668, 417

Gerssen, J., van der Marel, R. P., Gebhardt, K., et al. 2002, AJ, 124, 3270

Gezari, S. 2010, in IAU Symp. 267, Co-Evolution of Central Black Holes and Galaxies (Cambridge: Cambridge Univ. Press), 319

Gould, A. 2011, ApJ, 729, L23

Guillochon, J., Ramirez-Ruiz, E., Rosswog, S., \& Kasen, D. 2009, ApJ, 705, 844

Hamada, T., \& Salpeter, E. E. 1961, ApJ, 134, 683

Harris, W. E. 1991, ARA\&A, 29, 543

Henke, B. L., Gullikson, E. M., \& Davis, J. C. 1993, At. Data Nucl. Data Tables, 54,181

Herrmann, F., Hinder, I., Shoemaker, D., \& Laguna, P. 2007a, Class. Quantum Grav., 24, 33

Herrmann, F., Hinder, I., Shoemaker, D., Laguna, P., \& Matzner, R. A. 2007b, ApJ, 661, 430

Hinder, I., Vaishnav, B., Herrmann, F., Shoemaker, D. M., \& Laguna, P. 2008, Phys. Rev. D, 77, 081502

Husa, S., Hinder, I., \& Lechner, C. 2006, Comput. Phys. Commun., 174, 983

Irwin, J. A., Brink, T. G., Bregman, J. N., \& Roberts, T. P. 2010, ApJ, 712, L1

Kasen, D., \& Ramirez-Ruiz, E. 2009, ApJ, 714, 155

Katz, B., Budnik, R., \& Waxman, E. 2010, ApJ, 716, 781

Kawaguchi, T. 2003, ApJ, 593, 69

Kesden, M. 2011, Phys. Rev. D, 85, 024037

Kobayashi, S., Laguna, P., Phinney, E. S., \& Meszaros, P. 2004, ApJ, 615, 855

Komatsu, E., Smith, K. M., Dunkley, J., et al. 2011, ApJS, 192, 18

Krolik, J. H., \& Piran, T. 2011, ApJ, 743, 134

Laguna, P., Miller, W. A., \& Zurek, W. H. 1993a, ApJ, 404, 678

Laguna, P., Miller, W. A., Zurek, W. H., \& Davies, M. B. 1993b, ApJ, 410, L83

Lasota, J. P. 1994, in Proc. NATO Advanced Research Workshop, Theory of Accretion Disks, Garching, Germany, 1993 March 22-26, ed. W. J. Duschl, J. Frank, F. Meyer, E. Meyer-Hofmeister, \& W. M. Tscharnuter (NATO ASI Series C, Vol. 417; Dordrecht: Kluwer), 341

Lee, W. H., Ramirez-Ruiz, E., \& Page, D. 2005, ApJ, 632, 421

Lodato, G., King, A. R., \& Pringle, J. E. 2009, MNRAS, 392, 332

Loeb, A., \& Ulmer, A. 1997, ApJ, 489, 573

Löffler, F., Rezzolla, L., \& Ansorg, M. 2006, Phys. Rev. D, 74, 104018

Luminet, J.-P. 1985, Ann. Phys., 10, 101

Luminet, J.-P., \& Marck, J.-A. 1985, MNRAS, 212, 57

Luminet, J.-P., \& Pichon, B. 1989, A\&A, 209, 85

Magorrian, J., \& Tremaine, S. 1999, MNRAS, 309, 447

Mazzitelli, I., \& Dantona, F. 1986, ApJ, 311, 762

McClintock, J. E., Narayan, R., Davis, S. W., et al. 2011, Class. Quantum Grav., 28, 114009

McLaughlin, D. E. 1999, AJ, 117, 2398

Merritt, D., \& Poon, M. Y. 2004, ApJ, 606, 788

Mukai, K. 2000, PIMMS Version 3.0 Users' Guide (Greenbelt: NASA/GSFC)

Nauenberg, M. 1972, ApJ, 175, 417

Oppenheimer, J., \& Volkoff, G. 1939, Phys. Rev., 55, 374

Owocki, S. P., \& Gayley, K. G. 1997, in ASP Conf. Ser., 120, Luminous Blue Variables: Massive Stars in Transition, ed. A. Nota \& H. Lamers (San Francisco, CA: ASP), 121

Papaloizou, J. C. B., \& Pringle, J. E. 1983, MNRAS, 202, 1181

Phinney, E. S. 1989, in IAU Symp. 136, The Center of the Galaxy, ed. M. Morris (Cambridge: Cambridge Univ. Press), 543

Rees, M. J. 1988, Nature, 333, 523

Reid, I. N. 2005, ARA\&A, 43, 247

Rosswog, S., Ramirez-Ruiz, E., \& Hix, R. 2009, ApJ, 695, 404

Rybicki, G. B., \& Lightman, A. P. 1979, Radiative Processes in Astrophysics (New York: Wiley) 
Sadowski, A. 2009, ApJS, 183, 171

Sakimoto, P. J., \& Coroniti, F. V. 1981, ApJ, 247, 19

Schnetter, E., Hawley, S. H., \& Hawke, I. 2004, Class. Quantum Grav., 21, 1465

Sesana, A., Vecchio, A., Eracleous, M., \& Sigurdsson, S. 2008, MNRAS, 391, 718

Shakura, N. I., \& Sunyaev, R. A. 1973, A\&A, 24, 337

Shapiro, S. L., \& Teukolsky, S. A. 1986, Black Holes, White Dwarfs and Neutron Stars: The Physics of Compact Objects (New York: Wiley)

Shaviv, N. J. 2001, MNRAS, 326, 126

Shoemaker, D. 2009, Advanced LIGO Anticipated Sensitivity Curves LIGO Document T0900288-v3, https://dcc.ligo.org/cgi-bin/DocDB/ShowDocument? docid $=2974$
Stone, N., \& Loeb, A. 2012, Phys. Rev. Lett., 108, 061302

Strubbe, L. E., \& Quataert, E. 2009, MNRAS, 400, 2070

Thornburg, J. 2004, Class. Quantum Grav., 21, 743

Tolman, R. C. 1939, Phys. Rev., 55, 364

Tundo, E., Bernardi, M., Hyde, J. B., Sheth, R. K., \& Pizzella, A. 2007, ApJ, 663,53

Ulmer, A., Paczynski, B., \& Goodman, J. 1998, A\&A, 333, 379

Vaishnav, B., Hinder, I., Herrmann, F., \& Shoemaker, D. 2007, Phys. Rev. D, 76, 084020

Voges, W., Aschenbach, B., Boller, T., et al. 2000, IAU Circ., 7432, 1

Wang, J., \& Merritt, D. 2004, ApJ, 600, 149

York, J. W. 1979, in Sources of Gravitational Radiation, ed. L. L. Smarr (Cambridge: Cambridge Univ. Press), 83 